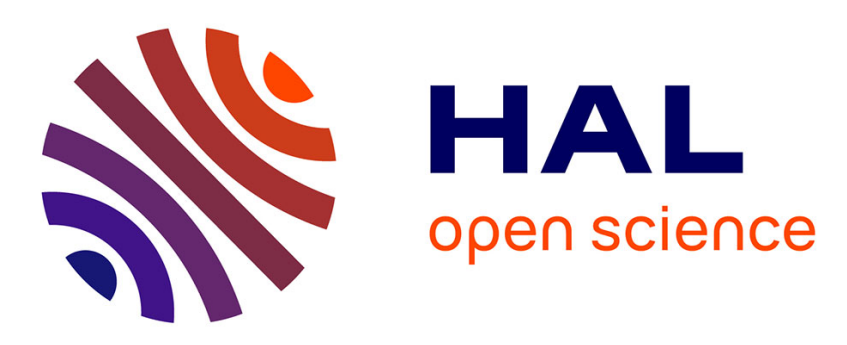

\title{
Multiple optical trapping and binding: new routes to self-assembly
}

T Cižmár, L C Dávila, Romero, K Dholakia, D L Andrews

\section{To cite this version:}

T Cižmár, L C Dávila, Romero, K Dholakia, D L Andrews. Multiple optical trapping and binding: new routes to self-assembly. Journal of Physics B: Atomic, Molecular and Optical Physics, 2010, 43 (10), pp.102001. 10.1088/0953-4075/43/10/102001 . hal-00569785

\section{HAL Id: hal-00569785 https://hal.science/hal-00569785}

Submitted on 25 Feb 2011

HAL is a multi-disciplinary open access archive for the deposit and dissemination of scientific research documents, whether they are published or not. The documents may come from teaching and research institutions in France or abroad, or from public or private research centers.
L'archive ouverte pluridisciplinaire HAL, est destinée au dépôt et à la diffusion de documents scientifiques de niveau recherche, publiés ou non, émanant des établissements d'enseignement et de recherche français ou étrangers, des laboratoires publics ou privés. 


\title{
Multiple optical trapping and binding: new routes to self- assembly
}

\author{
T. Cižmár ${ }^{2}$, L.C. Dávila Romero ${ }^{1}$, K. Dholakia ${ }^{2}$ and D.L. Andrews ${ }^{1}$ \\ ${ }^{1 .}$ University of Nanostructures and Photomolecular Systems, School of Chemical Sciences, \\ University of East Anglia, Norwich NR4 7TJ, United Kingdom \\ 2. SUPA, School of Physics and Astronomy, University of St Andrews, North Haugh, Fife, \\ KY16 9SS, United Kingdom
}

\begin{abstract}
$\underline{\text { Abstract }}$
The impact of optical forces in the physical and biological sciences now extends from familiar uses in trapping to more recent concepts of forces and torques enabling the manipulation of objects ranging in size from biological cells down to a single atom. These mechanical effects of optical fields have profound and far-reaching consequences, and attention is increasingly focused upon the opportunities for the non-contact assembly of particles into specific geometries. The present overview focuses on the two aspects of multiparticle trapping and optical binding. These can broadly be grouped as methods based on light-mediated inter-particle interactions, offering potential for the organisation of large numbers of micro- or nano-particles using optical forces alone.
\end{abstract}

\section{Introduction}

Few could have predicted the immense impact that optical forces have had in the physical and biological sciences. Whilst we are familiar with the uses of light for imaging and spectroscopy, the concept of light exerting a force by imparting the momentum of a photon may perhaps appear rather insignificant. However, at scales ranging from a single cell right down to that of a single atom, the mechanical effects of optical fields now have profound and far-reaching importance. In fact, the ideas embodied in this field have a history going back to the time of Kepler, four hundred years ago. In De Coriolis (1619), he published observations regarding the tails of comets pointing away from the sun at all points on their highly eccentric orbits. To explain the phenomenon, Kepler conceived the notion of some form of solar light pressure - a revolutionary proposition signifying the basis for the first ideas that light, despite its intangible nature, could exert a real mechanical force.

The arrival of the laser, with its capacity to deliver highly intense, directional and coherent radiation, rapidly opened up a range of new opportunities to optically manipulate small particles. Within the modern field of applications, there have been incredible advances at the atomic scale where the radiation pressure and dipole (or gradient) forces of light have been used to great effect. Radiation forces resulting from absorption and spontaneous emission cycles have been used to remove kinetic energy from atomic ensembles by repeated absorption and emission and appropriate internal atomic states, initiating what is termed laser cooling. The very low (and originally unexpected!) temperatures associated with this method led to the Nobel Prize in 1997 being awarded for these advances. The gradient force in itself provides a means to controllably guide and trap atoms, this force relying in particular upon absorption followed by stimulated emission. Powerful "sub-Doppler" cooling techniques have allowed scientists to access microkelvin temperatures for atomic ensembles, without the 
need for cryogenic apparatus. Combined with evaporative cooling techniques, this has ultimately paved the way for the realisation of a weakly interacting Bose-Einstein condensation and ultracold Fermi gases [1,2]. Crucially for the purposes of our discussion the forces of light and their impact upon the mechanical motions of a body is not restricted to the atomic scale: in fact, the very same types of optical force may trap and move objects that range in size from the nanoscale, right up to the size of a mammalian cell (a few tens of microns in diameter). This is the range of size scales that dominates the subject matter of the present article.

Nearly four decades ago, Arthur Ashkin [3] performed experiments upon micron-sized spheres with a visible (argon ion) laser. With a single, horizontally propagating laser beam he saw the microspheres aligning on the propagation axis, subsequently to be pushed along it, due to radiation pressure of the beam: this was the first observation of optical guiding. The addition of a second counter-propagating light beam (of equivalent optical power) created a radiation pressure force in the opposite direction. Under these conditions the guiding effect could be removed and instead the particle was held at rest between the two laser beams: the first optical trap was formed. This dual beam trapping geometry [3] was made more compact and practical with the use of single mode optical fibres over twenty years after the first observation [4]. Pertinent to this review, this setup has been central for the realisation of longitudinal optical binding $[5,6]$.

Following these counter-propagating beam studies, Ashkin [7] also investigated the stability of trapping of hollow and glass spheres with optical beams, balancing optical forces against gravity. In the case of hollow spheres annular light fields were used [8, 9]. In 1986 Ashkin et al. realized particle confinement with the single beam gradient trap (popularly known as optical tweezers) [10]. This is now the most accessible and ubiquitous method for applying optical forces as a means of moving microscopic particles. Optical tweezers have now been well-recognized as having the largest impact within the broader field of optical micromanipulation [11]. Whilst the technique is powerful, and important in providing a miniscule calibratable force transducer, a major impetus that has developed in the area has been to explore multiple trapping and inter-particle interactions.

Optical micromanipulation has seen a consistent and ever-increasing impact across all of the natural sciences, and one of the major topics of the last decade has been the use of creating a plurality of trap sites to trap multiple objects in pre-defined geometries: this is the area of multiple optical trapping. Related to this has been the emergence of the area of optical binding where optically modified particle-particle interactions dominate. Whilst there are several recent review papers in the general field of trapping [12-15], the present overview is distinguished by focusing on these two aspects of multi-particle trapping and optical binding. These can broadly be grouped as methods to understand light-mediated inter-particle interactions, offering the potential to organise large numbers of microparticles using optical force alone. In a combined sense, this is undoubtedly one of the most future important directions in the field, particularly from the viewpoint of the physical sciences.

The article is organised as follows: in the next section we describe the theoretical basis for single beam optical trapping, including a discussion of how the forces may be understood to operate at different size scales. We then progress to describing experiments related to multiple optical trapping, demonstrating why this is now such a significant issue. The majority of these experiments exhibit interesting arrays of microparticles but ignore the particle-particle interactions that lead to optical binding - which may play a dominant role in 
the system. Later parts of the article are devoted to this topic of optical binding, exploring the theoretical basis for the field and the experiments performed to date. Optical binding may complement and enhance multi-particle trapping to create more advanced self-assembled structures.

\section{Theoretical basis for single beam optical trapping}

In addition to techniques exploiting well-established radiation pressure effects, many methods have been developed to take advantage of high intensity coherent radiation having large field gradients, enabling the trapping and sorting of particles with continuous-wave laser beams [14] The single-beam trap exploits the highly inhomogeneous spatial field distribution of the beam, causing strong optical forces to act upon any particle near the focus, creating an attraction towards the highest intensity part of the field via the gradient force. We also of curse have scattering forces present in the system. At a point where these forces balance we will achieve a stable equilibrium which results in a "trap".

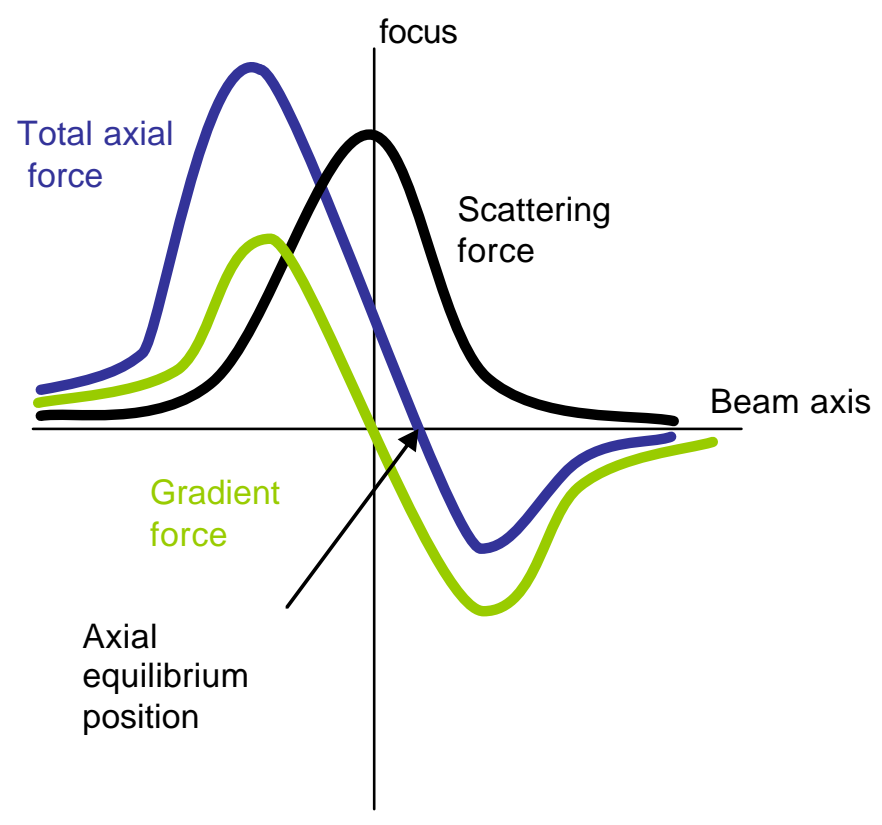

Fig. 1. Addition of scattering and gradient forces produces an axial position of equilibrium

When studying radiation pressure experimentally, the presence of thermal forces can obscure and destabilize trapping effects. These thermal forces result from temperature gradients in the medium surrounding the particles; caused by the absorption of radiation, they produce a thermal motion effect known as photophoresis [16]. A straightforward solution to such problems is the use of relatively transparent media and particles. 
The ratio of sizes between the small particles involved and the wavelength of the radiation determines the mechanistic regime under which the system must be considered; there are two important limiting cases. For dielectric particles whose radius is much smaller than the optical wavelength, each particle acts as a simple oscillating dipole, and the system is said to be in the Rayleigh regime. For cases where the particles are large compared with the wavelength, the geometric optics regime suffices to describe the phenomena of optical forces that result from refraction and reflection. The following sections focus on these two regimes.

\subsection{Rayleigh regime}

Single-beam intensity gradient traps were originally designed for application to Rayleigh particles, where the particle radius $r$ is much smaller than the wavelength $\lambda$ of the electromagnetic radiation. To implement an optical trap entails the use of a highly intense and strongly focused beam, and the trap stability exhibits a balance between the attractive forces due to the beam's gradients, forcing particles towards the narrow focus of the beam, and the repulsive scattering force acting away from the focus, in the direction of the throughput light. Within the dipole approximation, the force on a particle with subwavelength radius, due to its dynamic interaction with an electromagnetic wave, can be expressed simply by the Lorentz force,

$$
\mathbf{F}=(\mathbf{p} \cdot \nabla) \mathbf{E}+\dot{\mathbf{p}} \times \mathbf{B}
$$

where $\mathbf{E}$ and $\mathbf{B}$ are the electric and magnetic fields of the radiation, respectively; $\mathbf{p}$ is the particle's induced dipole moment and $\dot{\mathbf{p}}$ is its time derivative. The first term in equation (1), which arises due to the interaction of the induced dipole with the inhomogeneous field, delivers the gradient force. The second term delivers a force that fluctuates at the optical frequency and which, in terms of particle dynamics, can therefore be ignored.

The total Lorentz force, experienced by a particle in a medium of refractive index $n_{m}$ accommodates both a gradient and scattering force. In particular, when electromagnetic plane-waves are considered and a linear dipole response to the electric field is assumed, $\mathbf{p}=\alpha \mathbf{E}$ where $\alpha$ is the polarizability, and the total optical force is $[10,17-19]$

$$
\mathbf{F}=-\frac{n_{m}}{2} \alpha \nabla\left(E^{2}\right)+\frac{16 \pi^{4} \alpha^{2}|E|^{2}}{3 \varepsilon_{0} n_{m} \lambda^{4}} \hat{\mathbf{k}}
$$

where $\mathbf{k}$ is the radiation wave-vector, $\lambda$ its corresponding wavelength, $\varepsilon_{0}$ the vacuum permittivity and the effective particle polarizability for a homogeneous dielectric particle is

$$
\alpha=4 \pi \varepsilon_{0} n_{m}{ }^{2} r^{3}\left(\frac{m^{2}-1}{m^{2}+2}\right) .
$$

In equation (3), $m=n_{p} / n_{m}$, where $n_{p}$ is the refractive index of the particle. For finite radiative frequencies, particles will scatter and attenuate the electromagnetic radiation. Consequently a phenomenological correction to the polarizability expression (3) may be added in order to account for this effect [19]. However, where the dipole approximation is a good representation, this correction effect is in general minor. As particle size diminishes, the 
polarizability delivered by (3) progressively evolves towards the dynamic molecular polarizability whose dispersion properties can be determined by quantum electrodynamical calculation.

The threshold for axial stability of a single beam trap occurs when the ratio, between the backward axial gradient force and the forward scattering force, is unity. In particular, for a Gaussian beam of focal spot size $w_{0}$ at an axial position $z=\pi w_{0}^{2} / \sqrt{3} \lambda$, we have: [10]

$$
\text { ratio }=\frac{3 \sqrt{3}}{64 \pi^{5}} \frac{n_{m}^{2}}{\left(\frac{m^{2}-1}{m^{2}+2}\right)} \frac{\lambda^{5}}{r^{3} w_{0}^{2}} \geq 1
$$

Optical trapping under these conditions has been demonstrated in numerous experiments with latex and metal microparticles, their sizes ranging from tens to hundreds of nanometres. [2022]. It is interesting to comment on the exact role in optical trapping of the polarizability $\alpha$ and refractive index, issues of particular interest when considering metallic nanoparticles. In any material the polarizability depends on the electronic properties and the mutability of the charge distribution under the influence of the electromagnetic field. In general, the index of refraction is an effective medium concept based on a homogenization of the microscopic polarizability over the particle in question. This assumes that the constituent molecules or atoms of the material are all in the same average environment, equivalent to an infinitely extended bulk material.

In the case of dielectric nanoparticles this is a valid approach, as the local polarizability originates from bound electrons. Generally, the gradient force is proportional to the particle volume, and for this reason it is very difficult to trap inert dielectric nanometre-sized objects. Metallic particles, though, differ in several respects; per unit volume they present much larger polarizability values. To accurately model the optical forces exerted on metal nanoparticles it is necessary to consider the associated dispersion properties. Specifically, in the Drude model, the frequency-dependence of the polarizability given by (3) emerges from the formula $m^{2}=1-\left\{\omega_{p}^{2} /\left(\omega^{2}+i \gamma \omega\right)\right\}$, where $\omega_{p}$ is the plasmon frequency and $\gamma$ is the damping. It also becomes important to consider that the behaviour of the conduction electrons alters, when they are in close proximity to the metal surface. If the particle dimensions are smaller than the corresponding 'skin depth', the incident field interacts with the whole particle volume, resulting in a linear dependence between force and volume - the particle is rather transparent. However, for particle sizes greater than the skin depth, the field interacts only with a thin outer layer and the force varies as the square of the diameter.[2326]

\subsection{Ray optics regime}

The single-beam optical trap, first used for Rayleigh-size particles, was later found to be also a good instrument for trapping larger particles on the micron scale. Such traps are now used to manipulate a variety of biological systems, including living cells, where the best results are obtained with infrared traps, to reduce photodamage due to the trapping light [15]. Here, the focus of most studies is on particles sufficiently large for the propagation of light within them to follow the principles of simple ray optics. Calculations based on a model sphere can thus be used to describe the optical forces exerted by the scattering of light. Figure 2 [27], shows a 
qualitative representation of a dielectric particle trapped by a single optical beam. The totality of the beam can be decomposed into individual rays, each with an appropriate intensity, direction and polarisation, propagating in straight lines within uniform media of refractive index $n$. Each ray can change direction when it reflects or refracts, and it changes polarisation at dielectric interfaces according to the Fresnel equations. The refraction of light ray at the particle surfaces results in a transfer of momentum from the trapping laser to the particle; the rate of momentum delivery determines the overall trapping force.

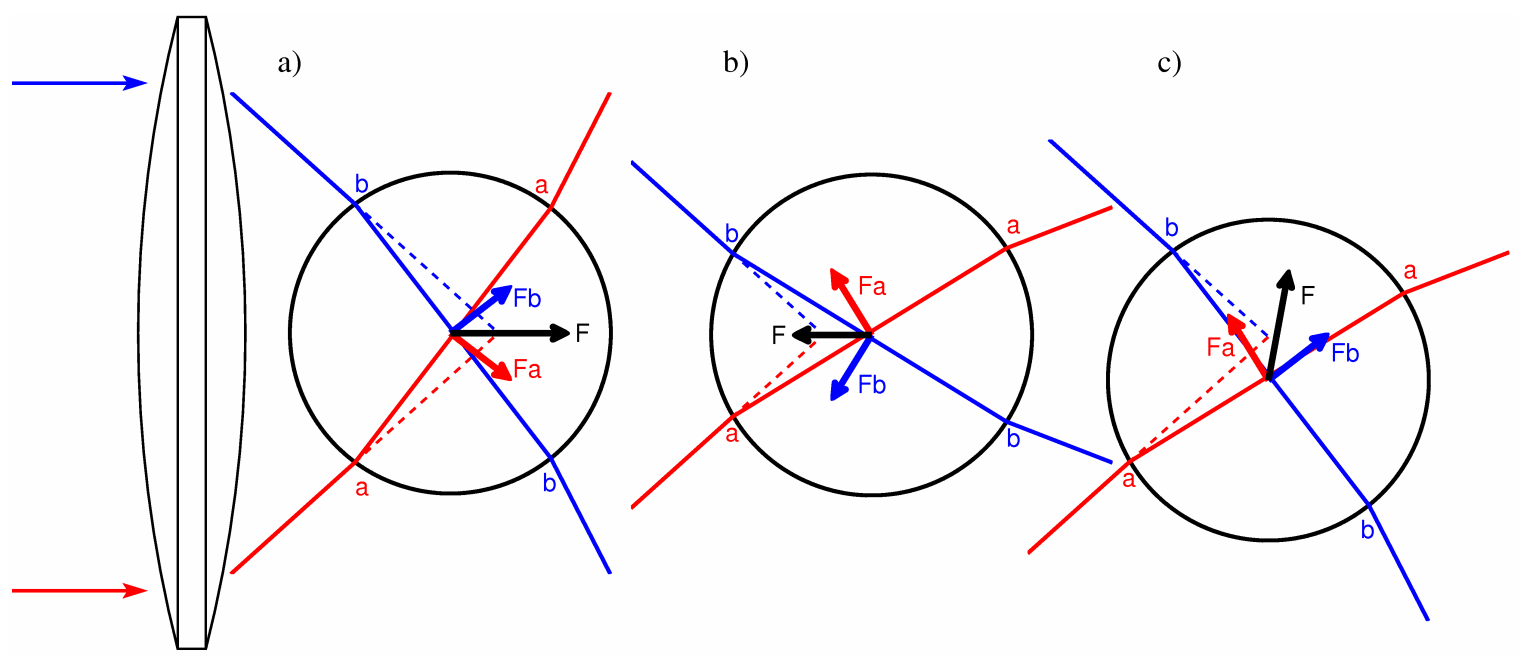

Fig 2: Qualitative view of the trapping of a dielectric sphere, showing that the refraction of a typical pair of rays $\mathrm{a}$ and $\mathrm{b}$ of the trapping beam result in forces, $F_{a}$ and $F_{b}$ respectively, whose sum is always restoring towards equilibrium for axial and transverse displacements of the sphere from the trap focus, located at the intersection of the dashed lines. The cases a) and b) show the axial restoring force acting on a particle that is axially displaced from the focal plane. Similarly c) shows the lateral restoring force upon the particle towards the beam focus.

The analysis is based on the calculation of forces due to individual rays of power $P$ and angle of ray incidence on the sphere surface $\theta$. The total force on a spherical particle comprises a sum of contributions due to reflected rays of power $P R$, and an infinite number of emergent refracted rays of successively decreasing power $P T^{2} R^{m}$, with $m=0,1,2, \ldots$, where the quantities $R$ and $T$ are the Fresnel reflection and transmission coefficients of the surface at incidence $\theta$. The result can be expressed as follows, in a form that exhibits a close relationship to the classical Maxwell-Bartoli relation; [28]

$$
|\mathbf{F}|=|\mathbf{Q}| \frac{n_{m} P}{c} .
$$

Here $n_{m}$ is the refractive index of the medium and the trapping efficiency $|\mathbf{Q}|$ is dependent on the Fresnel coefficients $R$ and $T$ - for a more detailed analysis see [27]. Accordingly the total force, equation (5), acting on the particle is described as a sum of forces $F_{a}$ and $F_{b}$ resulting from the refraction and convergence of the beam, on the simplifying assumption of zero surface reflection. Detailed calculations show that, for arbitrary displacements of the sphere's centre, the restoring force directs the sphere back to the focus, making the trap stable.

\subsection{Mie and Lorenz-Mie regime}


For particles whose linear dimensions approach or are equal to the optical wavelength, classical electrodynamics can be used to apply the conservation law for linear momentum in an optical field. Here the net optical force exerted on an arbitrary object is completely determined by Maxwell's stress tensor $\overrightarrow{\mathbf{T}}(\mathbf{r}, t),[29]$,

$$
\langle\mathbf{F}\rangle=\int_{S}\langle\overrightarrow{\mathbf{T}}(\mathbf{r}, t)\rangle \cdot \mathbf{n}(\mathbf{r}) d a
$$

where the angular brackets denote a time average, and the integral over $S$ signifies a closed surface enclosing the particle, with $\mathbf{n}(\mathbf{r})$ defining the outward unit vector normal to the integration surface. The optical force expressed in these terms is of general validity for an arbitrary (albeit rigid) body within the surface; it is entirely determined by the electric and magnetic fields at the surface. If the body deforms, additional electro- and magneto-strictive forces must be included. The fields used for the calculation of force using equation (6) are self-consistent fields, i.e. superpositions of the incident and scattered fields. If the media in which the dielectric particle resides is represented by an isotropic electric permittivity $\varepsilon_{m}$ and magnetic susceptibility $\mu_{m}$, then Maxwell's stress tensor has components given by:

$$
T_{i j}(\boldsymbol{r}, t)=\varepsilon_{m} E_{i} E_{j}+\mu_{m}^{-1} B_{1} B_{j}-\frac{1}{2}\left(\varepsilon_{m} E^{2}+\mu_{m}^{-1} B^{2}\right) \delta_{i j}
$$

Detailed calculations of this approach can be found in work by Barton et al. [30, 31]. The stress tensor can be calculated for a sphere with the help of Mie theory, considering all fields inside and outside of the sphere to be continuous at the surface.

A different approach to the Maxwell stress tensor has been described by Rohrbach [32, 33]. Here the approach is similar to that followed in the Rayleigh regime, where the optical force described in terms of the Lorentz expression, (1), is split into two components: the scattering force, and the gradient force. The advantage of this approach is that the particles can be of arbitrary shape, and the interaction can be determined for arbitrary modes of the electromagnetic field. However, whe re the particle size is similar or slightly smaller than the wavelength, the local structure of the electromagnetic fields invites attention.

\subsection{The counter-propagating dual beam trap}

Before considering the creation of arrays of multiple traps, it is appropriate to note the deployment of other single particle trapping geometries, beyond the standard optical tweezers. The major other single particle confinement technique has been a dual beam trap, as briefly described above, that uses two counter-propagating fields. As with all traps, it is the interplay between the gradient force and the scattering force that is key. The dual beam trap was revolutionised by Constable and colleagues in 1993 using a dual fibre geometry [4]. This is interesting as it makes the trap compatible with microfluidic channels, and it requires no high numerical aperture optics for operation - though of course such optics are desirable for viewing. This trap has a large catchment area and ability to distribute the optical force over substantial regions. This has led to the confinement of large cells: The dual beam system has proven interesting for combining trapping with other methods such as optical stretching and Raman spectroscopy[34, 35] and of interest for us the dual beam trap has been a key to major experiments in the geometry of longitudinal optical binding, as discussed later [6]. 


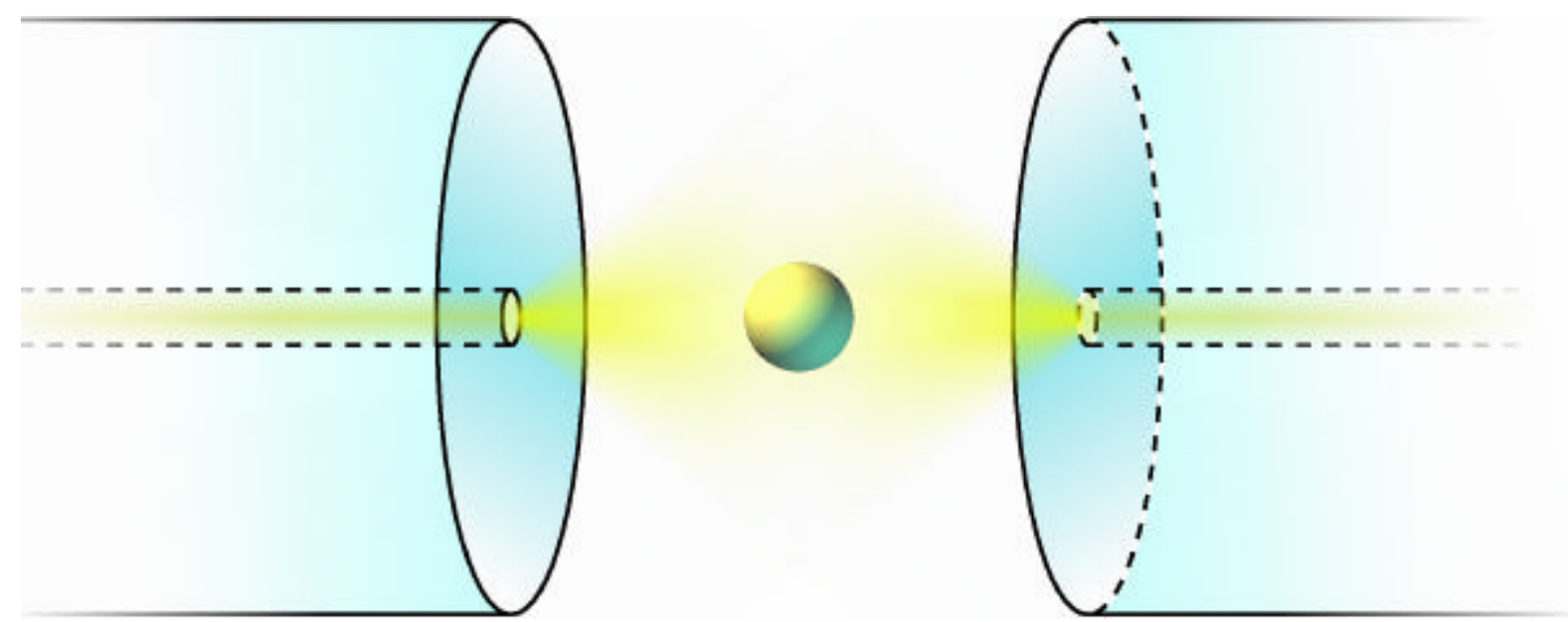

Fig 3: Diagram of a dual beam fibre trap geometry. A particle is kept between the fibres by gradient force in the lateral direction and in the axial direction it seeks the position where the scattering forces from both fibre generated beams are balanced.

\subsection{Traps for precision measurements}

It is worth briefly commenting here upon how optical tweezers are converted into measurement apparatus for macromolecules and cell biology. An optically trapped bead in a buffer medium (e.g. water) turns out to be a very good example of an over-damped oscillator with a rapid frequency roll-off of around $1 \mathrm{kHz}$ or so [15]. In this area of study, rather than trap any molecule directly (which would be hard due to its small size and polarisability) a bead is functionalised with surface chemistry (e.g. a biotin-streptavidin link) to tether it to the molecule of interest to the bead. To create a useable force transducer requires ready calibration of the system, and this may be achieved by use of fast position-detection system. Whilst the trapped object is held in a diffraction-limited beam, particle excursions are of course well below this distance. The trap creates a parabolic optical potential well. For small displacements away from trap centre, the force is directly proportional to displacement - a microscopic version of Hooke's Law. It is therefore possible to use high precision position sensitive detectors to calibrate the displacement and so record the trap stiffness. Whilst the relative force may be calculated, calibration via measurement of the Brownian motion is the technique of choice for absolute measurements. Here, either the bead position is recorded, temporal correlation measurements made, or one obtains the power spectrum of the bead locations, which can show a characteristic roll-off frequency. The accurate determination of trap position is achieved either via a quadrant photodiode detector (very high bandwidth, typ. $10 \mathrm{kHz}$ ) or fast camera [36]. It is important to place these studies in the context of multiple traps and binding: as we look ahead we see that positional analysis of multiple trap sites and bound particles will indeed pave the way for more elaborate studies in cell and molecular biology.

The most widely adopted procedure interprets the power spectrum of Brownian fluctuations in position of a trapped particle. This behaviour may be described by the Einstein-OrnsteinUhlenbeck theory of Brownian motion that leads to a Lorentzian power spectrum. In turn the Lorentzian form of the spectrum permits explicit analytical results for the values of fitted parameters as functions of the experimental power spectrum. The trap strength is consequently derived with a high precision that may be limited mainly by the calibration of the position detection system. Several papers elucidate the theory in this case $[37,38]$. 
In this manner, highly accurate measurements may be made: particular examples include the field of microrheology [39]. High resolution stepwise motion of macromolecules can be recorded. A good example is the motion of kinesin upon microtubules (in $\sim 8 \mathrm{~nm}$ steps) [40] the famous muscle contraction system: actin-myosin. Very recently, angstrom level displacement has been achieved with an ultra-stable apparatus to explore the key process of transcription where RNA polymerase (RNAP) moves processively along a DNA template, creating a complementary RNA [41].

\section{Experiments and technology for multiple traps}

\subsection{Introduction}

Single beam optical tweezers present an excellent tool for various biomedical, colloidal and fundamental studies. We first discuss the limitations of using a single Gaussian beam for a single optical trap: this will lead to an understanding of the appeal of multiple-trap arrays and the use of optical binding for larger scale particle organisation. Whilst powerfully effective, optical tweezers have some inherent limitations. Single beam tweezing uses a microscope objective lens and a zeroth order Gaussian laser beam. This results in an ellipsoidal volume at beam focus, which in turn constrains the ratio of trap stiffness in the lateral and axial directions. A single Gaussian beam thus does not lend itself to tailoring the trap (and thus its stiffness) in a three-dimensional space.

Furthermore, diffraction of the Gaussian beam potentially limits the types of applications, e.g. optical guiding or sorting of microparticles. In addition to lateral and axial motion, securing precise rotational control within optical tweezers opens up new possibilities in the domain of microrheology [42, 43]. This rotational control may be achieved with rotating light patterns, or the transfer of spin or orbital angular momentum to a given microparticle. A particle spinning at a constant rate reaches equilibrium related to the medium's viscosity, and so may used to measure a range of viscoelastic behaviour in different media. Spinning single or multiple particles can induce fluid pumping within a microfluidic environment; [4446] this may be employed for mixing small volumes of analyte. By increasing the plurality of optical trap sites and creating multiple traps we may implement different trapping experiments in parallel, or work with larger arrays of cells or colloids.

Importantly, an extended array of optical tweezers may be represented in terms of an optical potential energy landscape (a pattern of potential energy wells created by light). With suitably disposed beams, this can impose optical forces over a large region. Such landscapes can optically arrange microparticles or cells into two and three dimensional quasi-crystals. Furthermore, one can tune the inter-particle interaction in this multi-particle system by surface chemistry or judicious use of solvent. Such an assembled ensemble of particles allows for major studies in materials science and thermodynamics [47, 48]. In the following sections we explore in particular the topic of multiple trapping. With regard to the use of more exotic light fields (e.g. Bessel and Laguerre-Gaussian beams) we refer the reader elsewhere [12]. The community acknowledges that this topic holds great promise for future studies, extending the remit of optical trapping. For example, assembling a set of given microparticles into a predetermined geometry is of significance for materials science research. A wide range of innovative science may be performed with independent control 
over a given trap site and invoking large arrays of trap. Collective or co-operative effects between objects positioned within a potential landscape are also of interest: these can be dictated by the sample medium, particle surface chemistry or hydrodynamics for example. Dillen et al. discuss and emphasise how by, the self assembly of microparticles, [49] it is possible to study phase transitions (liquid-solid). These are just some of the reasons to explore techniques to generate extended trap arrays. In the next section we discuss how to make large trap arrays, concentrating on the two most powerful and widely used methods.

A plurality of discrete optical traps may be generated by a number of methods: a direct and straightforward way of obtaining multiple trapping sites is through the use of interference [50-52]. This naturally uses two or more beams, and may create 2D and 3D arrays of particles at will. Dynamic interference optical landscapes were used for long-distance delivery of objects in free space or near the surface [53, 54]. Exploiting the optical near-field is also another power method to obtain a large area self assembly of particles, given the large potential surface area over which particle may be organized [55-57]. Other methods include using arrays of diode lasers, particularly vertical-cavity surface-emitting laser (VCSELs), to generate 2D trap arrays [58-60]. However it is not trivial to generate appropriate variations in periodicity along a given dimension in any pattern, thus imposing some basic limitations. Therefore, in cases where fast and precise positioning of multiple optical traps at will is required, such geometries are not suitable. Much better results can be achieved by controllable splitting of a single trapping beam into a number of trapping positions. For a limited number of traps this can be achieved using beam-splitters or gratings [61]. However, for advanced experiments two technologies have come to the fore, namely the method of time-sharing a laser beam (typically acousto-optically) and generating multiple sites by wavefront shaping using dynamic diffractive optics techniques (typically in the form of a spatial light modulator).

\subsection{Time-sharing multiplexing of optical traps}

Optically trapped particles or cells are generally dispersed within a liquid medium (e.g. water or a buffer solution). The viscous nature of the medium means the trapped object behaves as a harmonic oscillator in a highly over-damped regime. Thanks to the slow diffusive rate of the particles we can afford to move a laser beam away from a given trap site and return it later if the time of absence is not too long. Once the trapping field is moved away from a particle's position, the particle is left free to diffuse due to Brownian motion. It can be assumed that the laser field revisits its original position and "re-confines" the particle within a very short time period (e.g. $<0.1$ millisecond). The diffusion distance $d$ of a particle in a given time $t$ may be determined by:

$$
d=\sqrt{2 \frac{k_{B} T}{6 \pi \eta r} t},
$$

where $k_{B}$ is the Boltzmann constant, $T$ is the absolute temperature, $\eta$ is the viscosity, $r$ is the radius of the particle and $t$ is the time during which the optical tweezing beam is removed. Hence, the smaller the particle, the larger the diffusion distance whilst the trap is absent. In this way it is possible to simultaneously trap multiple particles at each of the discrete sites arbitrarily displaced over a focal plane of a microscope objective, by rapidly time-sharing the laser field between each trapping site. This aspect of time-sharing, however, naturally leads to a maximum number of optical traps. Positioning of the trapping beam in a sample plane is 
typically provided by a beam-steering element placed to a conjugated plane with respect to the used microscope objective back aperture. A typical geometry for this time-sharing method is presented in figure 4 .

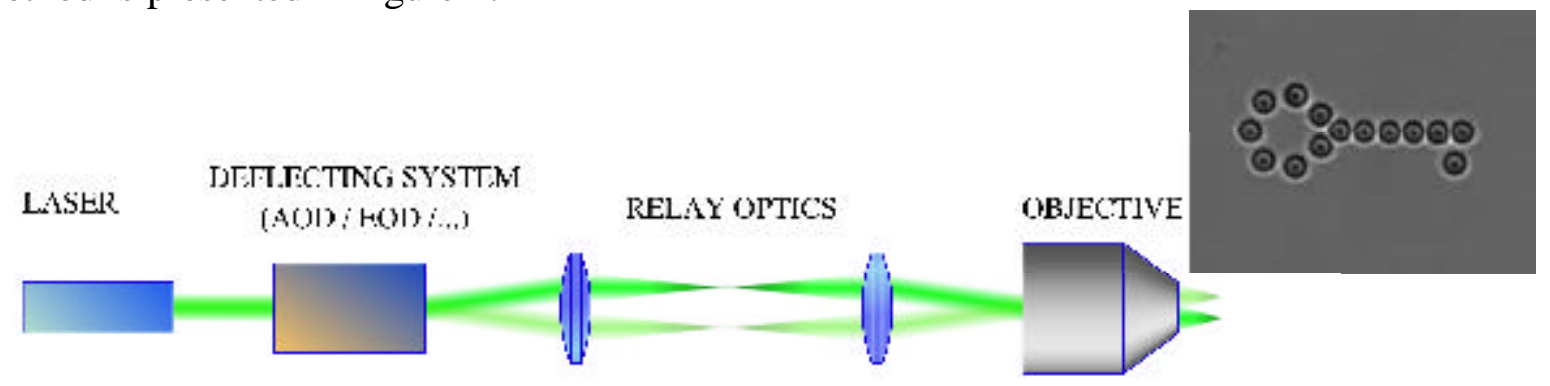

Fig 4: Generation of multiple optical traps using time-sharing method. One is able to send a laser beam light only to one trapping position at the time, however the ability of very fast switching between the trapping positions enables one to create up to several hundred stable time-shared optical traps in one axial plane. The beam-steering element (galvo/pezo-mirror, AOD or EOD) and the objective back apertures are placed at conjugated planes of a relay telescope.

The concept of a scanning trapping system was demonstrated by Sasaki et al. [62,9] by using a pair of fast scanning mirror driven by galvanometers. By scanning a single trap repeatedly along a specific path, microspheres were aligned into a designated 2-dimensional patterns. In this instance the group were able to hold metallic and low refractive index particles by rotating the incident light field in a circular path. Nowadays the systems can operate at timesharing rates of up to $2 \mathrm{kHz}$. Some improvements in resolution are seen with piezoelectric systems [63].

The most popular form of time-shared traps are generated by acousto-optic deflectors (AOD), as presented by Visscher et al. [64]. This device enables tuneable and highly precise beamsteering. The principle is based on the acousto-optic effect in an appropriate medium: a piezoelectric transducer generates an acoustic wave at radio frequencies (RF, tens of $\mathrm{MHz}$ ) propagating inside a transparent crystal (e.g. tellurium oxide). This induces a periodic modulation of a refractive index along one direction where a light beam propagating through diffracts similarly as it would from a phase grating. The steering angle is thus controlled by a frequency of the RF signal applied to the piezoelectric transducers, and the RF amplitude influences the intensity of the light in the $1^{\text {st }}$ diffraction order (the maximal $1^{\text {st }}$ order diffraction efficiency is typically around $70 \% ; 50 \%$ for 2 -axial steering). Using a pair of AODs aligned orthogonally with respect to one another along the propagation axis one can generate a number of optical traps, arbitrarily displaced over the focal plane, with timesharing rates of $\sim 10 \mathrm{kHz}$. The technical limitation of this method is mostly caused by reflections of the acoustic wave on the crystal walls and its unwanted persistence after the RF signal is changed. Naturally this technique is limited usually to multiparticle manipulation in a single lateral plane, but more elaborate optical schemes may generate more layers using this method [65].

In combination with a precise measurement of particle position, most frequently provided by a quadrant photodiode [66, 15], the geometry of optical tweezers with AOD-based timesharing has become a frequently used basis for sudies in molecular or cell biology. For example, Dame et al. [67] used an AOD based optical trapping system $\mathfrak{b}$ independently 
handle two DNA molecules, allowing the systematic investigation of protein-mediated DNA-DNA interactions. Guilford et al. [68] optimized particle position detection for AOD generated multiple time-shared traps that enabled the simultaneous observation of kinesinmediated displacements on two kinesin-coated optically trapped particles.

An alternative to AOD-based systems may be found in the use of electro-optic deflectors (EOD) [69] where a refractive index gradient of the medium (for steering) is achieved by applying an external electric field. The power efficiency and the switching rates are much higher; however, the range of deflection angles attainable is very small.

A quantitative description of typical parameters for each of the time-shared devices discussed in this section is shown the following table.

\begin{tabular}{|c|c|l|c|c|c|}
\hline Device & $\begin{array}{l}\text { Switching } \\
\text { rate }[\mathrm{kHz}]\end{array}$ & $\begin{array}{l}\text { Power } \\
\text { efficiency } \\
{[\%]}\end{array}$ & $\begin{array}{l}\text { Angular range } \\
\text { of deflection } \\
{[\mathrm{mrad}]}\end{array}$ & $\begin{array}{l}\text { Resolution } \\
{[\mu \mathrm{rad}]}\end{array}$ & Reference \\
\hline Galvo-mirror & 1 & $--*$ & 500 & 10 & {$[62,9]$} \\
\hline Piezo-mirror & 1 & $--*$ & 50 & 0.1 & {$[63]$} \\
\hline AOD & $10-50$ & 50 & 30 & $<1 \mathrm{~nm}^{* *}$ & {$[64]$} \\
\hline EOD & $>100$ & 80 & 1 & $<1 \mathrm{~nm} * *$ & {$[69]$} \\
\hline
\end{tabular}

\subsection{Holographic optical trapping}

Rather than time-sharing the light field between a number of trap sites, a natural inclination would be to actually distribute the light field to all desired trap sites simultaneously. A number of techniques may achieve this, the most powerful being the use of diffractive optical elements in either a static or dynamic form. In particular, a popular technique in the last decade has been the use of holography to create the desired optical potential energy landscape in the sample. In holography, the intensity and phase (complex amplitude) of an object is captured by the interference patterns between the light scattered of the object and a reference light field, and recorded upon a light sensitive medium in the form of diffractive element (hologram). Illuminating the hologram with the reference beam can therefore reveal a virtual image of the object. In holographic optical tweezers (HOT), however, the diffractive element is not recorded but the concept of digital holography is implemented: the diffractive element is designed by a computer and appropriate algorithm to achieve the desired field - no physical object is needed from the outset. In the most common geometry the diffractive element is placed at the Fourier plane (any plane conjugated with the back aperture of the trapping objective lens) where it modulates the spatial spectrum of the desired field. 


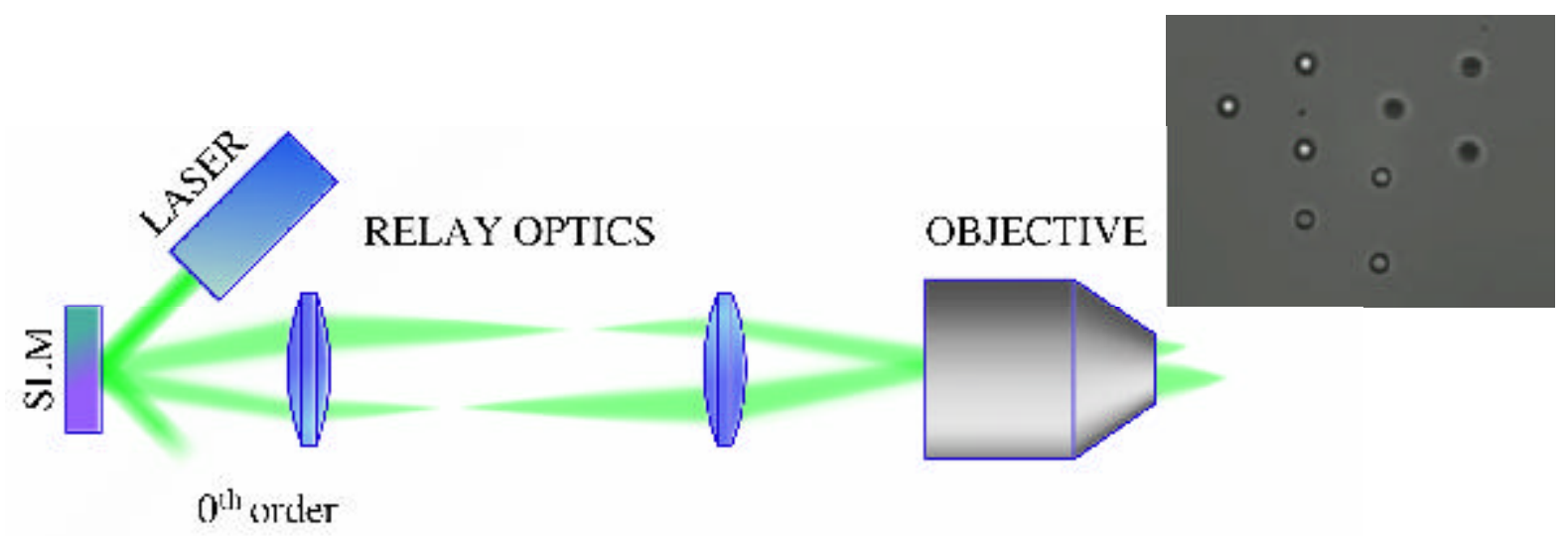

Fig. 5. Typical assembly of holographic optical tweezers. The $1^{\text {st }}$ diffractive order of the hologram on the SLM chip is imaged onto the objective back aperture by relay optics. The SLM hologram in this case is the Fourier conjugate of the optical pattern created at the sample plane.

The major advantages of such a scheme, when compared to time-sharing, are the possibilities to organize trap sites in three dimensions, to independently shape individual optical traps, remove aberrations, and to arbitrarily distribute power between them. One of the first implementations of holography in optical trapping was presented by Fournier et al. [70] who illuminated a binary hologram and generated self-images of the grating (Talbot images) in planes that are periodically positioned along the direction of propagation. Laguerre-Gaussian beams may have a phase singularity (vortex) at beam centre and an associated orbital angular momentum. They can be formed by a computer-generated hologram, and they have been used for trapping and rotation of a ceramic particle by He et al. [71, 72]. The computergenerated hologram can be imprinted by an etching procedure into a glass or plastic material. This then acts as a static diffractive element [73], or implemented with a liquid crystal display, spatial light modulator (SLM), typically an array of nematic liquid crystal pixels that are addressed either electrically or optically. The majority of the optical trapping applications is done by nematic phase SLMs, where one can shape the wavefront of the input field at will within the modulation bit depth (typically 256 levels), while the amplitude remains unchanged. The major advantage of this approach is the potential to rapidly reconfigure the imprinted phase modulation and so relocate the trapping sites. This attribute makes the use of SLM possibly the most promising technology of the upcoming prospects for multiple trapping and generation of sculptured light fields [74-87]. At present the resolution and refresh rate of nematic phase SLMs reaches the quality of LCD monitors and that makes their addressing extremely simple, typically using a DVI computer graphics card output. Ferroelectric SLMs, in contrast, can reach refresh rates of $\sim 10 \mathrm{kHz}$, but they are limited to only two levels of phase modulation [77].

The convenience of a hologram design algorithm for phase-only modulation relies on a particular application. For the case of a few optical traps, the most simple and frequently used approach is the algorithm of tilts and lenses, where the optical fields generating each individual optical trap are added, the resulting amplitude modulation is ignored, and only the phase modulation is used. This approach brings even higher power efficiency than exact complex modulation, but one creates a number of ghost orders that might impede the ideal performance of the trapping system. For a larger number of particles, and for all traps being placed on a single axial plane, much better light conversion (over 90\%) into the intended 
light pattern can be achieved using the Gerchberg-Saxton algorithm. Whyte and Courtial [88] showed that this technique can be extended to allow three-dimensional shaping of the trapping field. Since this is an iterative algorithm containing two Fourier transforms in each cycle, it can impose very high demands on computational power. Other algorithms might help to obviate this issue for the prise of significant power efficiency loss [83]. Finally we comment that one does not need to place the SLM in the Fourier plane, but indeed can use them in a "Fresnel" mode. The holographic read-out is performed in the off-axis Fresnel regime rather than in the typically used on-axis Fourier regime. The diffractive structure is computed as a Fresnel hologram. In this instance, after reflection at the SLM only the desired first diffraction order is guided to the input of an optical microscope, where the desired optical trapping field is created. The system is fast and suppresses undesired diffractive orders [89].

The use of the SLM in optical manipulation is not restricted to the standard Gaussian beams; any optical field can be multiplexed and positioned at will in three dimensions within a given volume. A good recent example is the direct multiplexing of a Bessel "non-diffracting" light beam in three dimensions using an axicon lens and SLM in tandem [90]. This particular beam has shown applications in optical sorting [91], nanopatterning [92] and cell transfection [90].

The generalized phase contrast technique is another method that uses SLMs in an optical trapping system. As an alternative to HOT, the SLM is used here as a phase element. The phase modulation encoded here is further converted to an amplitude modulation using a phase filter inside a $4 \mathrm{f}$ assembly of appropriate lenses [93]. The technique has analogies with the well known method of phase contrast imaging in microscopy. This conversion has a very high efficiency, and high computational power is no longer required - making it a very fast and potentially more amenable system for rapid motion and dynamical studies within optical traps. The system does not, however, allow a high focussing of the trapping beams, and so to achieve a stable 3-dimensional confinement one needs to control two counter-propagating beams from both sides of the sample chamber. This configuration has the advantages of longer working distances within the sample chamber, and potential ease of side viewing [94]. This has been successfully shown by Dam et al. and even realised in a compact, user-friendly geometry [95].

\section{Optical binding}

\subsection{Introduction}

The facility of optical trapping methods to isolate small numbers of microparticles in close proximity allows unprecedented experimental access to the study of inter-particle interactions. To set the scene for the intricate optically-induced forces that can operate between particles, and as a context for their better understanding, it is worth recalling the fundamental principles that operate at nanoscale levels. In the physical world we inhabit, chemically stable atoms and molecules are seldom found individually far apart; gases are the exception rather than the rule. Most matter exists in a condensed phase whose cohesion and integrity owe their origin to the fundamental interactions between the constituent particles of matter, primarily represented by dispersion forces. These are forces of attraction balance the short-range repulsions in regions of wavefunction overlap (as in the well-known LennardJones potential), supporting the formation of stable equilibrium structures. Dispersion 
(London) forces are open to a variety of interpretations, commonly being considered to arise from an electrostatic coupling between randomly fluctuating electric dipoles [96](p.633)

The notion that there could be a coupling between optically induced dipoles was first entertained by Thirunamachandran [97], in an elegant analysis based on quantum electrodynamical considerations. A subsequent, semiclassical analysis by Burns et al. [98] provided the first plots of the pair potential energy against centre separation for the simplest case of two identical, spherical particles - where particle separation is orthogonal to the Poynting vector. These graphs, exhibiting striking landscapes of rolling potential energy maxima and minima such as illustrated in Fig.6, instigated a rapid recognition of the enormous potential for practical applications, spurring on the first experiments. Indeed, the prospect for unprecedented precision in manipulating atoms and molecules was almost immediately flagged in an influential futurology of chemistry [99]. Later studies have shown that optically induced inter-particle forces offer a number of highly distinctive features which can be exploited for the controlled optical manipulation of matter. The first rigorous proof for the three dimensional stability of optical binding was given in work by $\mathrm{Ng}$ et al. [100] where they show that cluster consisting of three or more particles can be formed.

The terms 'optical binding' and 'optical matter', which have gained some currency for such forces, highlight the possibilities for a significant interplay with other interactions, such as chemical bonding and dispersion forces. Exploiting such interactions, new opportunities for creating optically ordered matter have already been demonstrated both theoretically and experimentally [50, 101-110]

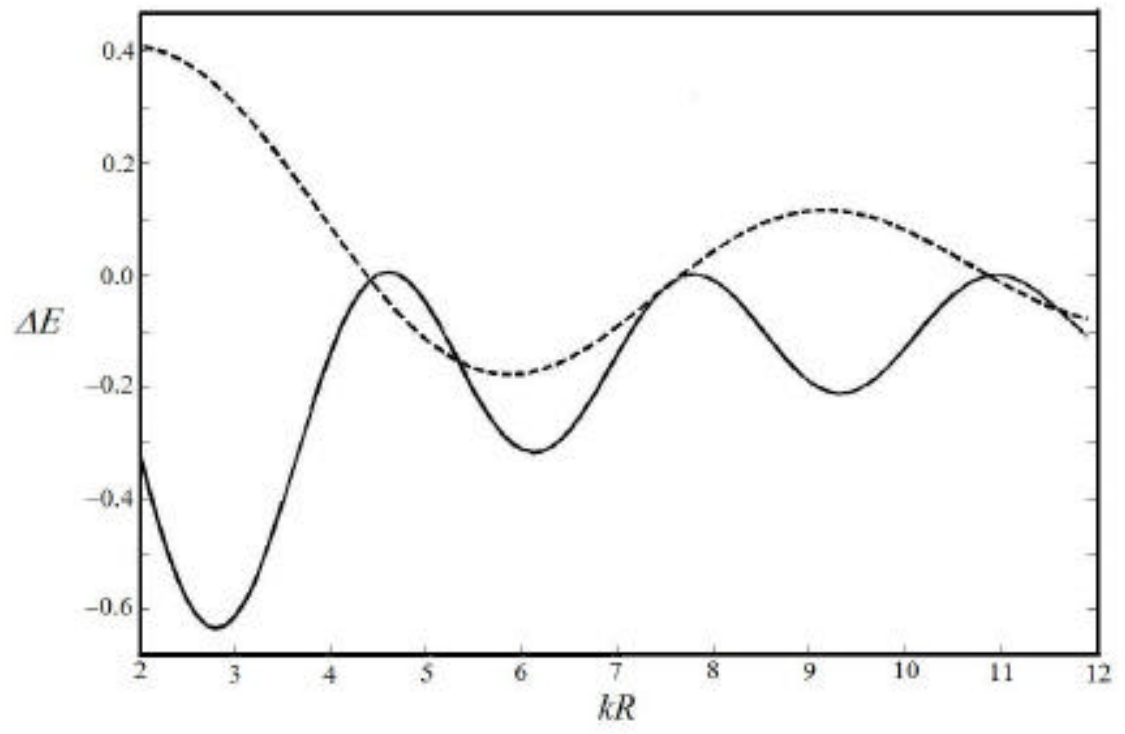

Fig 6.Dependence of optically-induced potential energy, for a pair of particles separated by distance $R$, plotted against $k R$, where $k=2 \pi \lambda$ and $\lambda$ is the laser wavelength. The solid line shows the case where the inter-particle axis is aligned with the propagation wave-vector direction $\mathbf{R} \square \mathbf{k}$, while the dashed line is the case where $\mathbf{R} \perp \mathbf{k}$ (figure by J. Rodíguez).

In the semiclassical depiction, the oscillatory electric field of monochromatic radiation produces motions in the charge distributions of the particles it encounters. Such motions lead to corresponding oscillating electric dipoles, whose phase is determined by that of the radiation at each particle. For two particles in sufficiently close proximity, the interactions 
between their oscillating dipoles is evidently subject to the relative phase of the optical field at the two locations; $\mathbf{i}$ is also subject also to the sharp decline of such an interaction with distance. From such a physical picture one can correctly surmise the physical existence of an optically induced force that exhibits a strongly damped oscillatory dependence on the separation of the particle pair. The result is not quite as simple as one might expect, however, even in the case of two spherical particles, because the electric field produced by an oscillating dipole has a relatively complex dependence on displacement $\mathbf{R}$ and orientation of the source dipole $\mu$, as delivered by the following equation; [29] ( p.411)

$$
\mathbf{E}=k^{2}(\hat{\mathbf{R}} \times \mu) \times \hat{\mathbf{R}} \frac{e^{\mathrm{i} k R}}{4 \pi \varepsilon_{0} R}+[3 \hat{\mathbf{R}}(\hat{\mathbf{R}} \cdot \mu)-\mu]\left(\frac{1}{4 \pi \varepsilon_{0} R^{3}}-\frac{\mathrm{i} k}{4 \pi \varepsilon_{0} R^{2}}\right) e^{\mathrm{i} k R}
$$

in which $k=\omega / c$ is the magnitude of the wave-vector $(2 \pi / \lambda)$ for an optical wavelength $\lambda$. In a medium of refractive index $n(\omega)$, equation (9) is modified by the replacement of each $k$ by $n(\omega) k$ leading to potential energy minima whose locations depend on the dispersion properties of the medium. While the above expression is fully consistent with the retarded near-field [111] inferred from an analysis based on quantum electrodynamics (QED), the latter theory affords deeper insights into the mechanisms at work in optical binding. The following QED development of theory not only affords a reliable route to results of general applicability, it illuminates the underlying relationship to other multi-centre interactions - in particular, dispersion forces. In the detail of the ensuing results, several facets of optical binding emerge that have yet to be tested experimentally, such as the detailed dependence on optical polarization, the behaviour of non-spherical particles, and the influence of deploying wide-bandwidth radiation.

\subsection{Quantum electrodynamical derivation}

To establish the QED representation of optical binding, it is helpful to recall how this theory addresses the normal dispersion interaction between particles. Here, calculations are performed on a system state where both the particles and the radiation field are in the ground state. This system state couples with other short-lived states in which the electromagnetic field has a non-zero occupation number for one or more radiation modes, signifying the presence of virtual photons. The dispersion interaction emerges from a perturbative calculation based on an exchange of two virtual photons, each created at one particle and annihilated at the other. These two quanta of light may (but need not) overlap in time as they propagate between the two units. Cast in such terms, the theory delivers a result - the Casimir-Polder formula - valid for all distances, correctly accounting for the retardation which leads to a long-range $R^{-7}$ asymptote dependence on the pair separation $R$ [112], [113] (p. 54) [114-118].

While this interpretation lends a fresh perspective to the physics involved in the more familiar $R^{-6}$ van der Waals interaction - the attractive part of the Lennard-Jones potential [119] (p.29) - it also strongly suggests that other effects may be manifest when the system state has an occupied radiation mode, i.e. when there is a throughput of electromagnetic radiation. Indeed it is the same, fourth order of perturbation theory that gives the leading result, and the optically conferred pair energies prove to exhibit a linear dependence on the photon number of the occupied mode. Cast in terms of experimental quantities, this is manifest as an energy shift $\Delta E_{\text {ind }}$, linearly proportional to the irradiance. The corresponding 
laser-induced coupling forces emerge from the potential energy result, as the spatial derivative.

QED delivers a result that is applicable to optical fields with arbitrary polarisation and beam geometry [120-122]. For the usual case of plane-polarized light the following result can be derived for the optically induced inter-particle potential energy, produced by a beam of irradiance $I$ acting on two particles $A$ and $B$;

$$
\Delta E_{i n d}(\mathbf{k}, \mathbf{R})=\left(\frac{2 I}{\varepsilon_{0} c}\right) e_{i} e_{l} \operatorname{Re}\left[\alpha_{i j}^{A}(k) V_{j k}(k, \mathbf{R}) \alpha_{k l}^{B}(k)\right] \cos (\mathbf{k} \cdot \mathbf{R}),
$$

where $\mathbf{R}$ is the separation between the particles, $\mathbf{R}_{B}-\mathbf{R}_{A}, e_{i}$ is the $i^{\text {th }}$ component of the laser polarization vector, $\alpha_{i j}^{\xi}(k) \equiv \alpha_{i j}^{\xi}(-k ; k)$ is the dynamic polarizability and $V_{j k}(k, \mathbf{R})$ the retarded resonance dipole-dipole interaction tensor [123];

$$
V_{i j}(k, \mathbf{R})=\frac{\exp (i k R)}{4 \pi \varepsilon_{0} R^{3}}\left[(1-i k R)\left(\delta_{i j}-3 \hat{R}_{i} \hat{R}_{j}\right)-(k R)^{2}\left(\delta_{i j}-\hat{R}_{i} \hat{R}_{j}\right)\right]
$$

In fact, the familiar dispersion interaction is a spontaneous, vacuum field component of the non-resonant optically-induced coupling. [124, 125] It is another example of direct link between QED and classical results - the same equation describes the field propagation between two induced dipoles (dyadic Green's function). The only other terms to arise in this order of perturbation theory, which involve static dipoles, are normally disregarded. Since the orientation of a free pair of particles with respect to a throughput beam is arbitrary, it is expedient to introduce a refe rence frame. As depicted in Fig. 7, we choose to define the direction of the electric field vector $\mathbf{e}$ as the $x$-axis, also denoting the common plane that contains the inter-particle vector $\mathbf{R}$ and the electric field vector as $(x, z)$. To represent the angular disposition we define as $\phi$ the angle subtended by $\mathbf{R}$ on to the electric field of the laser radiation; $\zeta$ designates the angle between the plane containing these vectors and the optical wave-vector $\mathbf{k}$. With spherical particles we then find;

$$
\begin{aligned}
\Delta E=\left(\frac{I(c k)}{2 \pi c \varepsilon_{0}^{2} R^{3}}\right) \operatorname{Re}\left[\alpha^{2}(-k ; k) \cos (k R \sin \phi \cos \varsigma)\right. \\
\times\left\{\left((1-\mathrm{i} k R)\left(1-3 \cos ^{2} \phi\right)-k^{2} R^{2} \sin ^{2} \phi\right\} \exp (\mathrm{i} k R)\right],
\end{aligned}
$$




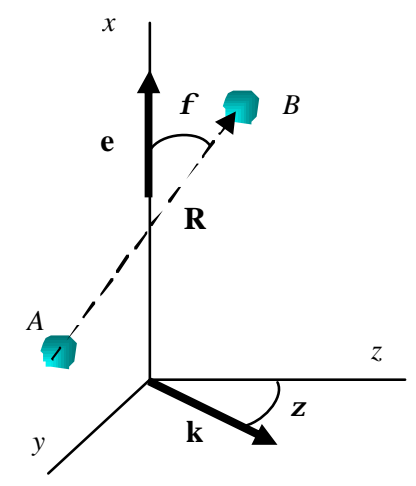

Fig 7. Particle pair orientation for optical binding (see text).

For example, for the commonly employed longitudinal binding geometry $\mathbf{k} \square \mathbf{R}$ we have $\phi=\pi / 2$ and $\zeta=0$, hence;

$$
\Delta E=\left(\frac{I(c k)}{2 \pi c \varepsilon_{0}^{2} R^{3}}\right) \alpha^{2}(-k ; k) \cos k R\left\{\left(1-k^{2} R^{2}\right) \cos k R+k R \sin k R\right\} .
$$

When the pair of particles is aligned with the polarization vector, equation (13) delivers a result whose graph exhibits the familiar $R$-dependence [104]. This result (alone) is independent of $\zeta$. For the angle dependence in general, it is a trivial matter to prove that, in the near-zone, the isotropic average of the optically induced pair energy vanishes. Nonetheless at all distances the energy landscape is decidedly anisotropic, and must therefore generate both positive and negative forces.

Extending the range of applications for the QED theory, calculations have also been performed for non-spherical particles - where the parameter space is substantially larger. One important case is where the particles have cylindrical symmetry, their polarizability being characterised by one transverse and one longitudinal component. The results, for cylindrical particles in various configurations, have been applied to single-walled carbon nanotubes. These nanoparticles are of interest not only for their intrinsic properties and applications but, since they are strongly polarizable species, they afford ideal opportunities to exploit the quadratic dependence on polarizability in the potential energy equations. Assuming that the $\alpha_{\perp}$ and $\alpha_{\square}$ values are consistent with the corresponding static polarizabilities, then for nanotubes $200 \mathrm{~nm}$ in length and $0.4 \mathrm{~nm}$ in radius, separated by a distance $R=2 \mathrm{~nm}$, and with off-resonant optical radiation at an incident intensity $I=1 \times 10^{16}$ $\mathrm{W} \mathrm{m}^{-2}$, the forces range between $10^{-12}$ and $10^{-5} \mathrm{~N}$, according to the geometry [126]. The results demonstrate that laser-induced forces can be either positive or negative according to conditions; use of the term 'binding' force in various papers [102, 104] is potentially misleading, though ingrained. To quantify the forces it might prove possible to deploy atomic force microscopy - a technique already associated with carbon nanotubes.[127] Significantly, the forces lie in a range indicating realistic possibilities for the nanomanipulation of carbon nanotubes, through laser optomechanical control. Other theoretical studies using Foldy-Lax multiple scattering of optical trapping and binding of cylindrical particles have been carried out by Grzegorczyk et al. [128, 129]. 


\subsection{Molecular aggregates}

To consider optical binding between molecular aggregates, each formed of $p$ molecules or optical centres, we need to entertain an effective polarizability of the aggregate given by the following expression [130];

$$
\chi_{i j}^{(\zeta)}=\sum_{\xi}^{p} \alpha_{\lambda \mu}^{(\xi)} I_{i \lambda}^{(\xi)} I_{j \mu}^{(\xi)} \exp \left(i \mathbf{k} . \Delta \mathbf{R}_{\xi}\right),
$$

where the $I_{\lambda i}^{(\xi)}$ are direction cosines relating the space-fixed to the molecule-fixed axes. The relative position of molecule $\xi$, within the aggregate $\zeta$., is given by $\Delta \mathbf{R}_{\xi}$, see Fig.8. The aggregates can be considered as mesoscopically disordered material, within which local domains possess particular ordered structures. The optically induced potential takes the form:

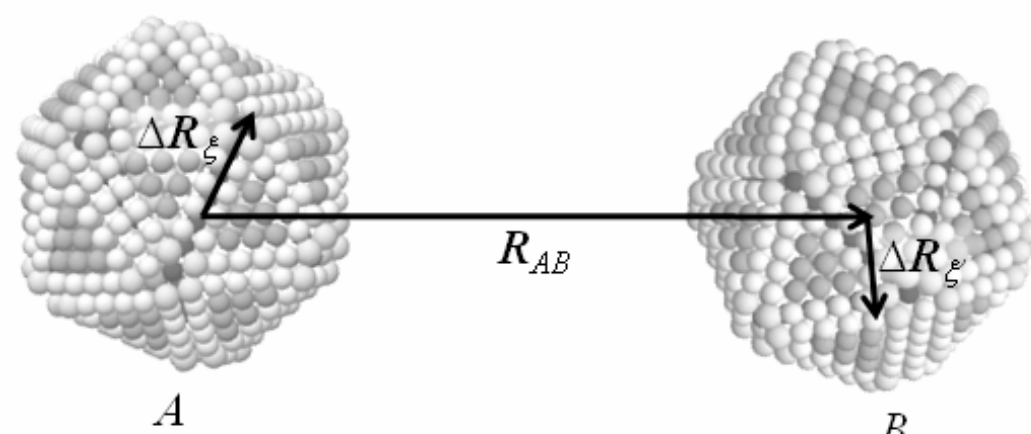

Fig 8. Molecular aggregates $\mathrm{A}$ and $\mathrm{B}$ separated by a distance $\mathbf{R}_{\mathrm{AB}}$

$$
\Delta E_{\text {ind }}^{\text {TOTAL }}\left(k, \mathbf{R}_{B A}\right)=\left(\frac{2 I \alpha^{2}}{\varepsilon_{0} c}\right) \operatorname{Re}\left(V_{x x}\left(k, \mathbf{R}_{B A}\right)\right) \operatorname{Re}\left[\sum_{\xi, \xi^{\prime}}^{p, p^{\prime}} \exp \left(i \mathbf{k} \cdot \Delta \mathbf{R}_{\xi, \xi^{\prime}}\right) \exp \left(-i \mathbf{k} \cdot \mathbf{R}_{B A}\right)\right]
$$

where $\Delta \mathbf{R}_{\xi, \xi^{\prime}} \equiv \Delta \mathbf{R}_{\xi}-\Delta \mathbf{R}_{\xi^{\prime}} \quad$ It has been shown [125] that the result carries a linear contribution which is present for particles of any size, persisting for large particles wherein $\Delta \mathbf{R}_{\xi, \xi^{\prime}}$ can be orders of magnitude larger than the optical wavelength $\lambda$, such as the case of micro-particles (see for example ref. [131]). However for nanoparticles where $\Delta \mathbf{R}_{\xi, \xi^{\prime}} \square \lambda$, there is a weighting factor that is quadratic in the number of molecules $p$. In the limiting case, by an analysis similar to that given in ref [132], the sum over molecules $\xi$ in the susceptibility may be approximated by a continuous integral and the optically induced energy of interaction between two spherical aggregates of radius $r$ emerges as follows; 


$$
\begin{aligned}
\Delta E & =\left(\frac{I \alpha^{2} \rho^{2}}{\varepsilon_{0} c k^{6}}\right)(\sin k r-k r \cos k r)^{2} \operatorname{Re}\left[V_{x x}(k, \mathbf{R})\right] \cos \left(k R_{y}\right) \\
& \equiv \Delta E_{0} \frac{\rho^{2}}{k^{6}}(\sin k r-k r \cos k r)^{2},
\end{aligned}
$$

where $\rho$ is the number density of molecules. Representing the dependence on $\rho$ in this manner demonstrates that optical binding, in aggregates comprising relatively few, high volume particles, will be less significant than in other aggregates of the same size, comprising a larger number of low-volume species. [133]

To complete the representation of a standard trapping environment, account should be taken of the dielectric influence of the medium in which particles are suspended. It has previously been shown, see for example [134-136], that the relative values of the refractive index between the particles and the surrounding medium significantly influences optical binding phenomena, modifying the positions of stability. With the incorporation of appropriate Lorenz field factors, the dependence on $k R$ changes to a dependence on $n(c k) k R$, where the multiplier is the complex refractive index of the medium supporting the particles. Most experiments are conducted with wavelengths at which the system is optically transparent, well away from any resonance. Then, the dielectric effect of the medium is equivalent to a simple scaling of the particle separations by the index $n$, i.e. the given results can be regarded as establishing the coordinates of energy minima on a scale where distance values are given by $R / n$.

\subsection{Potential energy landscapes}

As the distance between any particle pair increases, the optically induced force rapidly overtakes in magnitude the more sharply distance-dependent Casimir force. [136] Over short distances a convenient basis for comparison with the latter interaction is the dimensionless parameter $\hbar c^{2} k / I R^{3}$ ( $\hbar c^{2} k^{3} / I R^{5}$ in the long-range, $\left.k R » 1\right)$. With a wavelength of $355 \mathrm{~nm}$ and a modest irradiance of $10^{12} \mathrm{~W} \mathrm{~m}^{-2}$, the optically induced interaction typically exceeds the Casimir force at all distances beyond about $55 \mathrm{~nm}$, subject to the pair orientation. Although detailed force fields are derivable, they can also be inferred from the potential energy landscape, mapped from expression (12), and pictorial representations of the latter, scalar fields, are also visually less cluttered. When the optical input has a constant intensity, (or, if pulsed radiation is used, the throughput has a repetition interval that is short compared to the particle diffusion times), optical binding forces will operate to move particles to separations related to the locality of different minima in the energy shift landscapes, depending on the values of the salient geometric parameters [137].

Results from typical calculations are shown in Fig. 9, where angles $\phi$ and $\zeta$ are as defined in Fig7. On each of these plots, with a distance abscissa and an angle ordinate, local potential energy gradients are depicted by vector arrows; projections of these vectors on the horizontal axis thus denote forces directed towards local energy minima, those on the vertical scale corresponding torques. Similar graphs can be constructed to exhibit the dependence on the angular disposition of the wave-vector. 

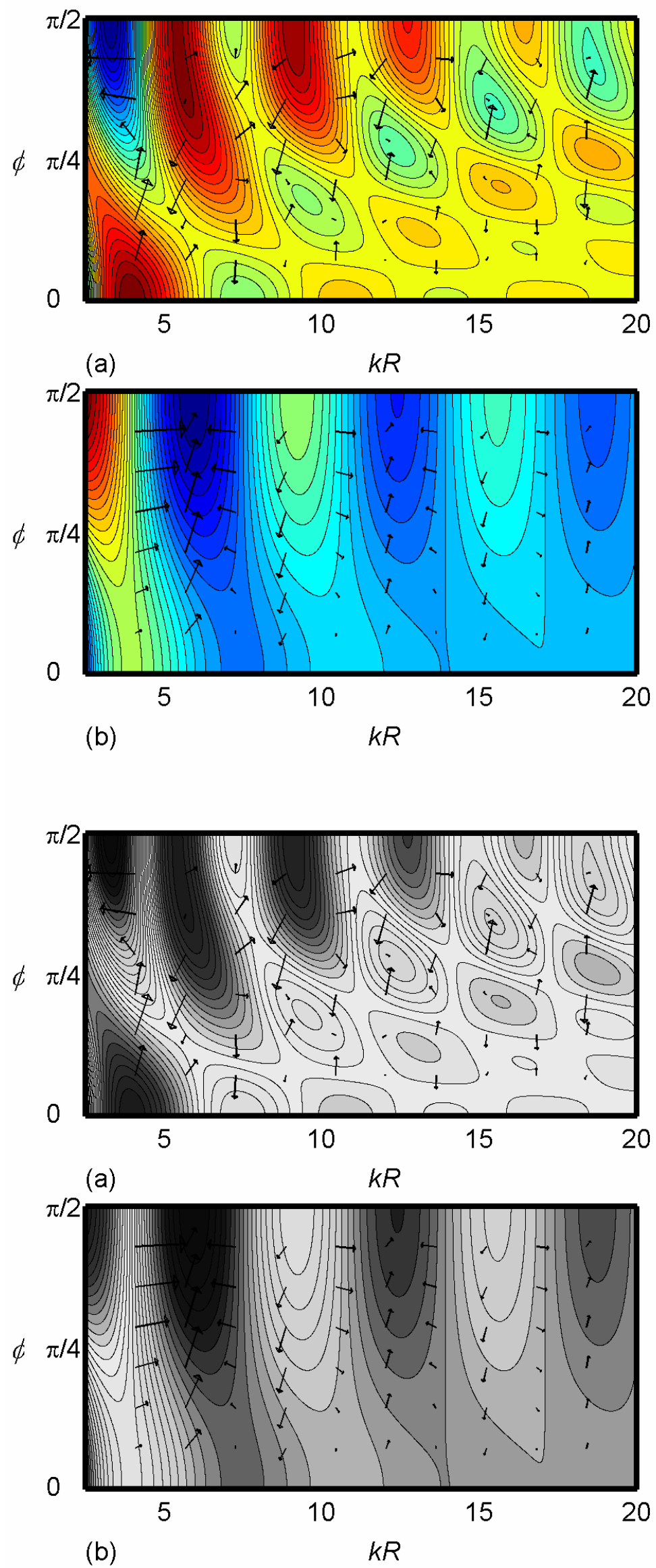
Fig.9 Contour maps of optically induced pair energy. Plots of $\Delta E$ as a function of $\phi$ and $k R$ : (a) $\zeta=0$; (b) $\zeta=\pi / 2$. The variation of $\Delta E$ with $k R$ along the abscissa, $\phi=0$, shows its first two maxima at $k R \sim 4.0,10.5$, the first (non-proximal) minimum, at $k R$ $\sim$ 7.5. The horizontal scale typically spans distances $R$ of several hundred nanometres, depending on the value of $k$ (see text). The units of the colour scale are $2 l k^{3} /\left(4 \pi \varepsilon_{o}{ }^{2} c\right)$. The arrows indicate the forces and torques that particle B experiences, according to its position. [136] [Color version for web: greyscale for printed version] Copyright (2008) Elsevier.

In each landscape, local minima distinguish optical binding configurations. The contours intersect the abscissa scale orthogonally, reflecting an even dependence on each angular variable; the variation in the domain $\{(\phi, \zeta)$ with $\phi, \zeta \in(0, \pi / 2)\}$ is notionally revealed by unfolding along the distance axis. The physical significance is that a system whose $(k R, \zeta, \phi)$ configuration has $\phi=0$ or $\zeta=0$, but not situated at a local minimum, is always subject to a force drawing it towards a neighbouring minimum without change of orientation. For the same reason, there is no torque when $\phi=\pi / 2$ or $\zeta=\pi / 2$. Most systems in an arbitrary configuration will be subject to both forces and torques. Fig. 9(a) illustrates the emergence of potential energy features supporting longer-range order, such as columnar patterns of alternating minima and maxima at constant $k R$, conducive to the formation of ring structures.

\subsection{Broadband radiation}

Recent theoretical research has shown that particle arrays whose formation is optically induced can be micromanipulated, assembled and dissassembled with precision by a secondary field [138]. Further control over the morphology of optically fabricated arrays can be achieved using continuum light.[124] For convenience the spectral irradiance of broad continuum light may be assumed to have Lorentzian form:

$$
S(\omega)=\frac{K}{\left(\omega_{0}-\omega\right)^{2}+\gamma^{2}},
$$

where $\omega_{0}$ determines the centre frequency; the parameter $K$ determines the maximum and $\gamma$ the width of the function. Each of the interacting pair of particles is assumed to comprise molecules with a two-level response dominated by the ground state, $|0\rangle$, and one excited state, $|r\rangle$; consequently the particle susceptibilities are given by:

$$
\chi^{(A)}(\omega)=\chi^{(B)}(\omega)=\frac{2 N}{3 \hbar} \frac{\omega_{r 0}\left|\mu^{r 0}\right|^{2}}{\varepsilon\left(\omega_{r 0}^{2}-\omega^{2}\right)},
$$

where $N$ is the number of optical centres per particle; $\hbar \omega_{r 0}$ is the difference of energy between the states $|0\rangle$ and $|r\rangle ; \mu^{r 0}=\langle r|\mu| 0\rangle$ is the transition moment associated with the 
electronic transition $|r\rangle \rightarrow|0\rangle$. Expression (18) is closely related to the molecular aggregates discussed in Subsection 4.3. To avoid absorption and attendant thermal effects, and to observe the dependence of the inter-particle potential energy surfaces on spectrally different forms of irradiance, part of the light tailored with a long-pass filter, whose effect is illustrated in Fig. 10. Under these conditions, and for simplicity ignoring the dispersive effects of a support medium, the inter-particle potential energy for each particle pair can be written as follows:[124]

$$
\begin{aligned}
& \left\langle\Delta E_{\text {avg }}\right\rangle= \\
& \operatorname{Re}\left[\frac{4 K N^{2}\left|\mu^{r 0}\right|^{4} \omega_{r 0}^{2}}{9 c \hbar^{2} \varepsilon_{0}} \int_{0}^{\omega_{1}} \frac{1}{\left(\omega_{r 0}{ }^{2}-\omega^{2}\right)^{2}} \frac{V_{x x}(\omega / c, \mathbf{R})}{\left(\omega_{0}-\omega\right)^{2}+\gamma^{2}} \cos \left(\frac{\omega\left(\mathbf{R}_{A B}\right)_{z}}{c}\right) d \omega\right] .
\end{aligned}
$$

where $\left(\mathbf{R}_{B A}\right)_{z}$ is the $z$-component of the separation vector $\mathbf{R}_{B A}$. It is then possible to explore the effect of choosing different spectral intervals by using different pass filters (hence different values of $\omega_{1}$ ). The graphs in Fig. 11 show the optically induced inter-particle potential along the Poynting vector of the beam for different values of $\omega_{1}$. The results reveal that, by increasing the region of the spectrum interacting with the particles, the magnitude of the binding energy is significantly increased as shown in the first three graphs; this is a simple consequence of the corresponding increase in irradiance. More significantly, the sequences of minima within each such curve form a series in which each is separated from the next by about the same distance, a condition that favours the formation of particle chains [139]. With a change of $\omega_{1}$, the locations of all the minima in every graph are displaced in a positive or negative sense in identical proportion. This suggests that any variation in the position of the first minimum is indicative of a corresponding change in the length of particle chains along the Poynting vector. The dependence on optical frequency of the location of the first potential energy minimum reveals that optically induced chains may be controllably extended or contracted by filtering appropriate frequency regions of the continuum input radiation.

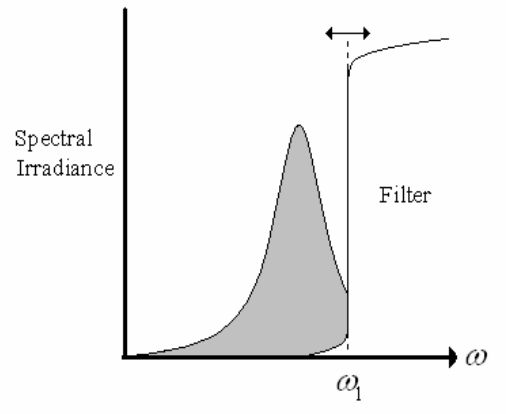

Fig. 10. Spectral irradiance of light, with Lorentzian spectrum, transmitted through a longwavelength pass filter. 


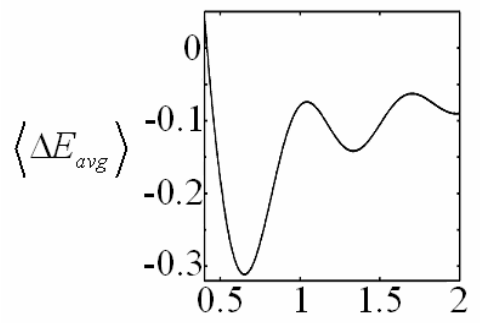

(a) $R_{z}$

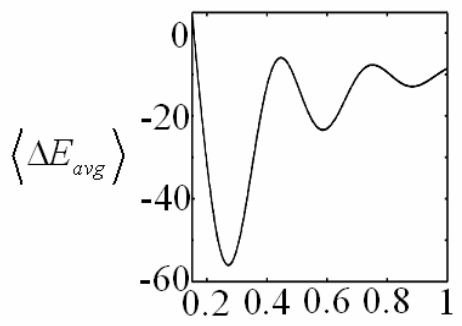

(c) $R_{z}$

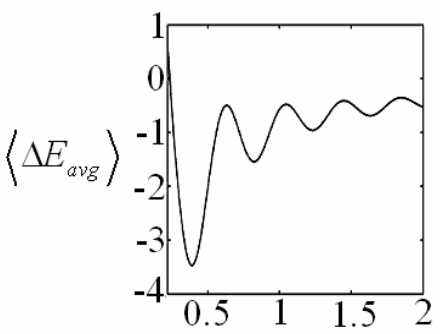

(b) $\quad R_{z}$

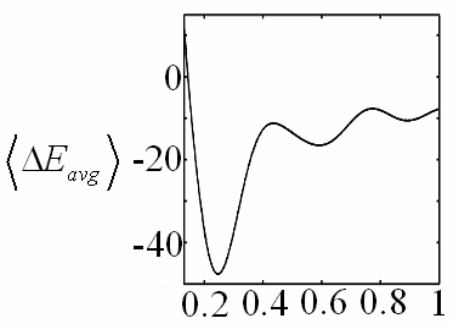

(d) $\quad R_{z}$

Fig 11. Optically induced inter-particle potential given by equation (11) for $\omega_{0}=10 \gamma$, $\omega_{r 0}=20 \gamma$ and for (a) $\omega_{1}-5 n(\mathrm{~h}) \cdots-8, \quad(r) \omega_{1}=12 \gamma \quad$ (d) $\omega_{1}=15 \gamma$. The units of energy and distance are $400 K N^{2}\left|\mu^{r 0}\right|^{4} /\left(c \varepsilon_{o} \hbar^{2}\right)$ and $c / \gamma$, respectively. The Poynting vector is along the $z$ axis, the polarization is along the $x$ axis and, $R_{z}$ the separation of particles along the $z$ axis. .

\section{Multiple particle optical binding}

\subsection{Optically fabricated particle assemblies}

Although optically induced pair forces fall away rapidly with distance, they are clearly dominated by nearest-neighbour interactions, so that it is possible to deduce the patterning of a particle assembly on the basis of pair interactions. From expressions (10) and (11), optically induced energy shift contour plots can be obtained, giving detailed information about the location of the system's stability points; see Fig. 13. In this figure the abscissa scale representing distance is cast in terms of the dimensionless parameter $k R$; starting at a non-zero value to avoid non-physical divergences that would imply overlap of the particles (in other words, the separation $R$ must be larger than the diameter of the particles involved). Additionally, these contour plots show that the derivatives of the energy with respect to $\zeta$ are zero at $\zeta=0$ or $\pi / 2$, independently of the value of $\phi$, indicating that no torques are present. Particles positioned elsewhere will experience forces and torques directed towards the nearest points of stability (represented by arrows in Fig.9). It can be observed that for $\zeta=\pi / 2$ (the propagation vector impinging transversely on to the pair, see Fig. 7) the only minima are found at $\phi=\pi / 2$. Naturally, when the pair is aligned with the polarization vector $\mathbf{e}$, the energy shift is independent of $\zeta$. Plots such as those exhibited are essentially templates from which the energy landscape for any larger number of particles can be ascertained.

The occurrence of multiple local minima and maxima in the energy landscapes indicates that a variety of assemblies may be possible; the probabilities of formation depend on the size and 
shape of the potential landscape concavities, determined by a sum of all pair interaction energies. The simplest assemblies are chain structures - linear distributions of particles, for which the angles $\phi$ and $\zeta$ (for polarized light) or $\gamma^{*}$ (non-polarized) have the same values for every A-B pair. If all such particles are identical, a regular one-dimensional lattice can form when they are all intercepted by light of the same intensity. This lattice will be based on a repeated superposition of the energy landscape, displaced by multiples of the distance corresponding to one particular minimum in the surface - usually the closest. A minor degree of stabilization or destabilization by the interaction between non-neighbouring particles might be present, modifying the stability of the linear assembly; an ideal case, with enhanced stability, arises when the relative dispositions of closest and non-closest particle pairs are simultaneously close to the locations of different energy minima. In all configurations the energy shift associated with interactions between next-nearest neighbours is about half of that between neighbouring particles, (essentially a consequence of the $R^{-1}$ energy shift dependence for large $k R$ ), consolidating the stability of the linear configuration [140].

Many more complex structures are also possible. For example with reference to Fig. 9(a), for polarized laser radiation, it is evident that with there is an energy minimum close to $\phi=\pi / 4$ (also $-\pi / 4,3 \pi / 4,-3 \pi / 4$ ) for $k R=12.5$, suggesting the possible formation of an assembly of four particles placed approximately on the vertices of a square, orthogonal to the beam axis. The occurrence of several minima for a fixed angle $\zeta$, as also shown in Fig. 9(a), further suggests the possible formation of variety of ring structures.

Such studies are valid in the Mie size regime, i.e. where the sphere diameter exceeds the wavelength, and input fields are well approximated as paraxial. Considering particles of like dimensions, Chaumet and Nieto-Vesperinas [104] have derived results both for isolated spheres, and for spheres near a surface. In the former case they have found that inter-particle forces depend significantly on the polarization and wavelength of the incident light, and the particle size. Furthermore, $\mathrm{Ng}$ and Chan [100] have determined the equilibrium positions in an array of evenly spaced particles, aligned in parallel with the wave-vector of the optical input.

\subsection{Three or more particles}

The energetics associated with the arrangements of more than two particles can now be explored by placing particles in the minima of a suitable template that optimizes the pair interactions. [141, 125, 138, 133] The two-particle landscape that is physically favored provides a sensible basis for determining the optically induced potential energy resulting from placement of one or more additional particles on the $(y, z)$ plane, as shown in Fig. 13. Contour maps of the energy surfaces of three interacting particles, as a function of the coordinates of one of the particles, are shown in Fig. 14. In contrast to earlier potential energy landscapes mapped to illustrate and differentiate forces and torques, these figures are plotted on a pair of Cartesian axes to clarify the relative positioning of configurations with more than two particles. As the number of particles increases, there is a dramatic rise in the number of stable, physically realistic arrays. When the fixed displacement vector is longitudinal to the direction of propagation of the particles, and $k R=3$ as in Fig. 14(a), the

\footnotetext{
* The angle $\gamma$ is defined as the angle between the wave-vector $\mathbf{k}$ and the separation vector $\mathbf{R}$.
} 
most significant minima are given the sequence $\left(k R_{x}, k R_{y}, k R_{z}\right)=(0,3 n, 0)$ for $n=2,3,4 \ldots$ with an energy that is inversely proportional to $n$; a longitudinal chain is the most stable configuration, as may also be observed in Fig. 14(b) when the magnitude of this separation is $k R=6$. When the displacement between the two fixed particles is transverse to the propagation direction, as in Fig. 14(c), for example, there is a sequence of potential energy minima at approximately the positions $(0,3 n, 3)$ for $n=1,2,3 \ldots$ with the lowest of them for $n=2$; another significant minimum may be observed close to the position $(0,8,3)$. When the fixed displacement vector is given by $(0,8.6,3)$, as in Fig. $14(d)$, there are two important symmetrically equivalent stability points at approximately $(0,2,3)$ and $(0,7,0)$. By extending the analysis is extended to four or more particles, it becomes evident that a variety of 2-dimensional arrays may be optically fabricated in a plane perpendicular to the polarization of the laser beam.
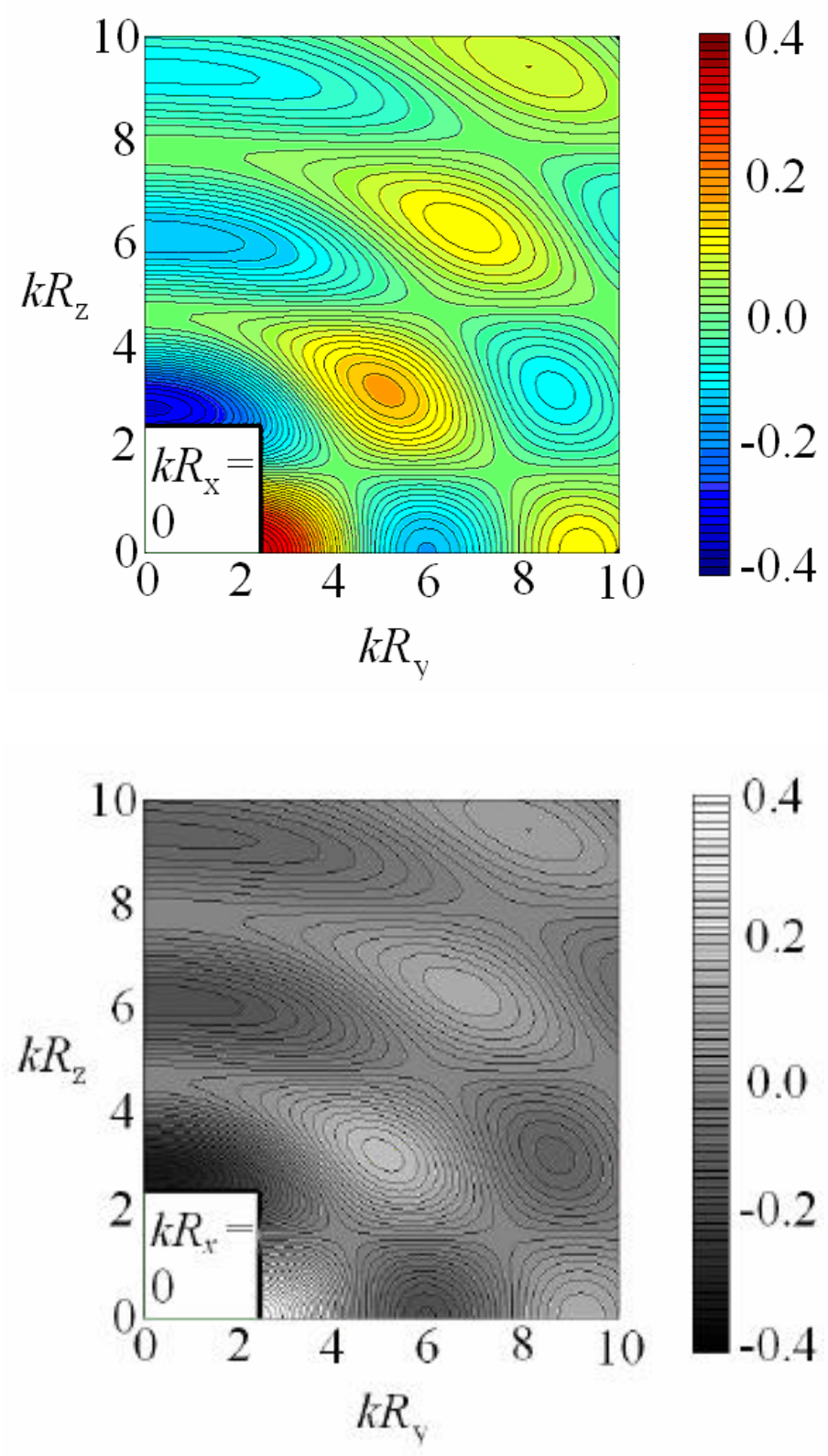

Fig 13. Optically induced inter-particle energy surface on the $(y, z)$ plane of Fig 7 . The scale is in $2 I \alpha^{2} k^{3} /\left(\varepsilon_{o} c\right)$ units. Field polarization directed along the $x$-axis, wave-vector 
on the $z$-axis. P. [Color version for web: greyscale for printed version] Copyright (2008) by the American Physical Society.

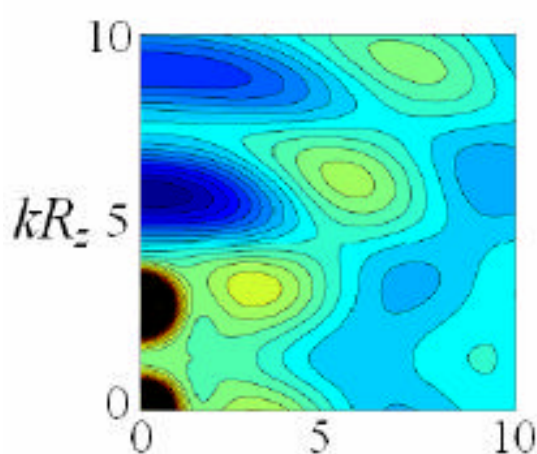

(a) $\quad k R_{1}$

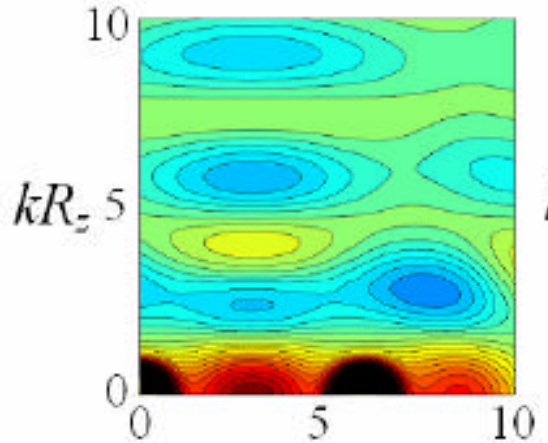

(c) $k R_{\text {. }}$

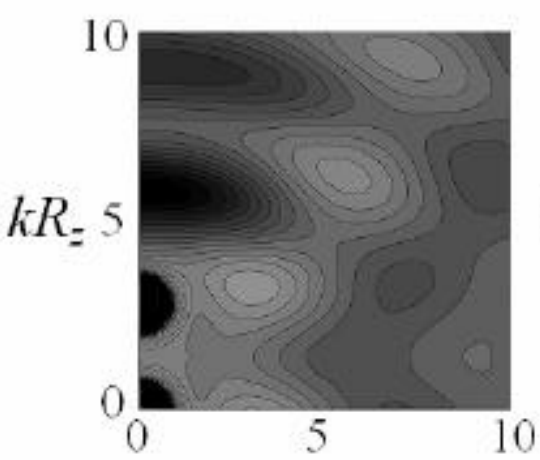

(a) $k R_{v}$

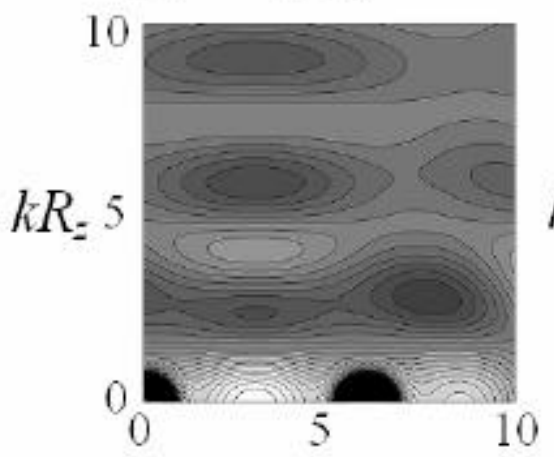

(c) $k R_{v}$

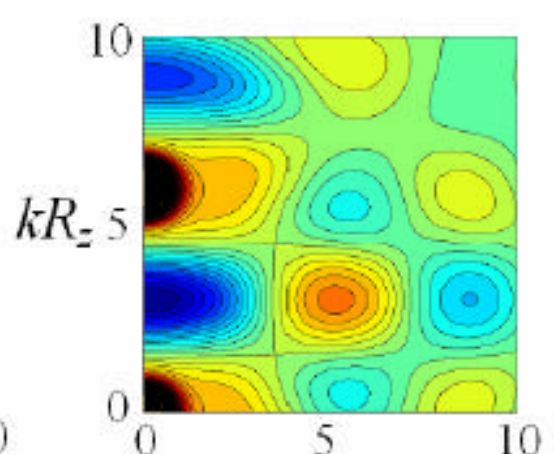

(b) $\quad k R_{v}$

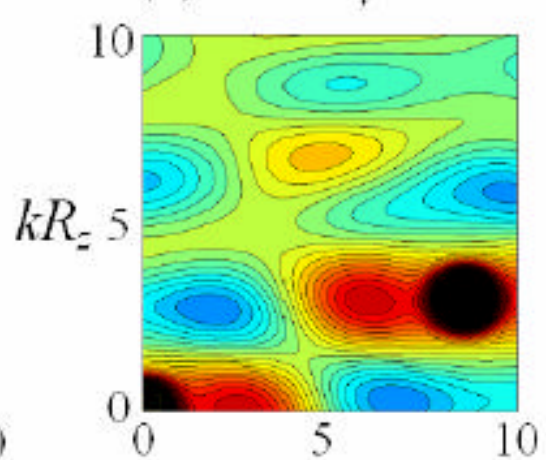

(d) $\quad k R_{v}$
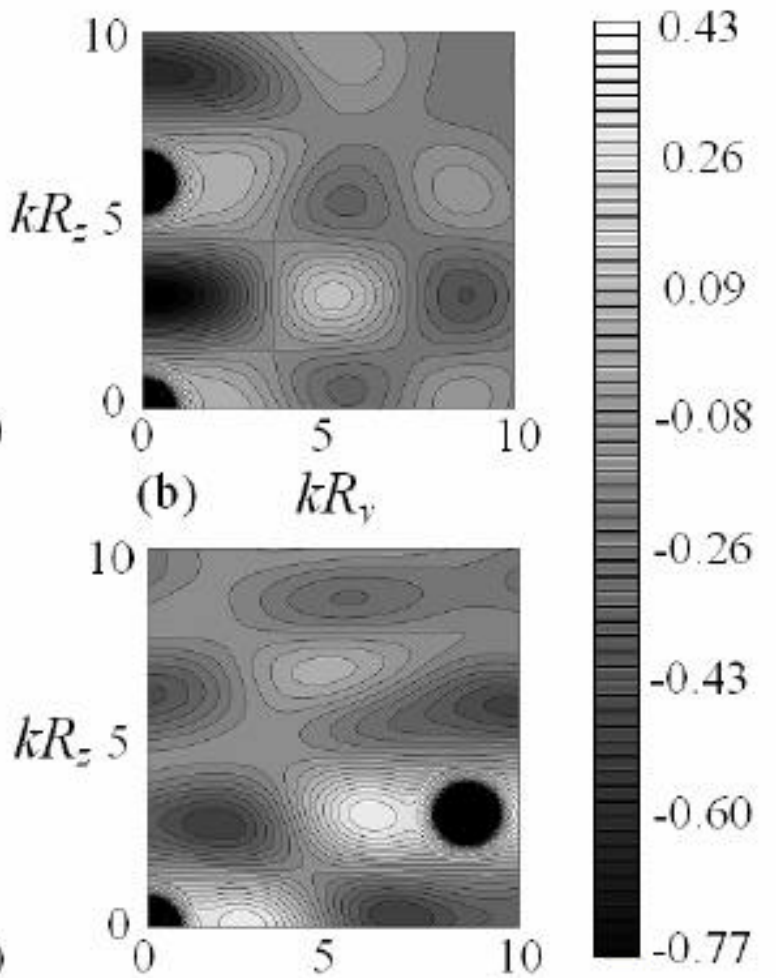

(d) $k R_{v}$

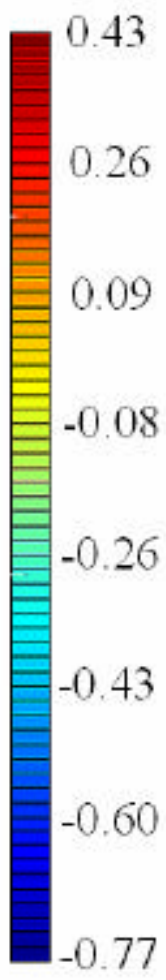

(b)

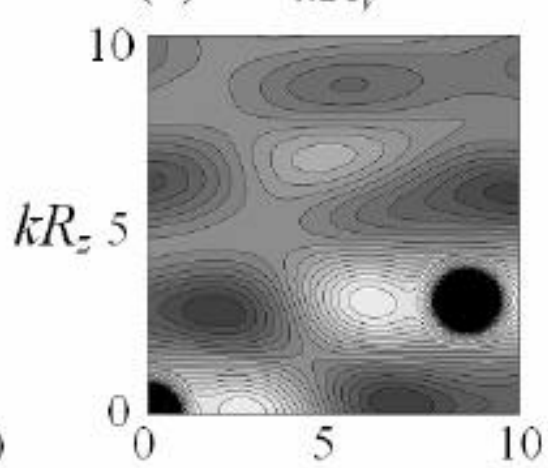

$-0.77$ 
Fig. 14. Optically induced potential energy landscapes for three identical interacting particles as a function of the vector positions of one of them, when the other two are located, respectively, at the origin and at: $(a)(0,0,3) ;(b)(0,0,6) ;(c)(0,6,0)$; (d) $(0,8.6,3)$. Black circular shapes represent local divergences in energy shift in the proximity of the fixed particles. Scale, polarization and wave-vector as in Fig. 13 [Color version for web: greyscale for printed version] Copyright (2008) by the American Physical Society

\subsection{Structured light}

One of the most rapidly developing areas of modern optics concerns the production and application of structured beams [142], for example Laguerre-Gaussian (LG) vortex beams produced with the use of bifurcated gratings. The associated introduction of optical phase discontinuities is typically manifest in nodal axes, planes and surfaces in the beam intensity profile. Correspondingly, such beams display characteristically structured regions of high intensity where particle trapping can occur. A classic example is the case of an optical vortex with one radial node at the beam centre, a common feature of a 'twisted' LG beam with arbitrary $l$ and $p=0$ (these two indices serve to designate a specific modal structure, i.e. $l$ defines the azimuthal mode while $p$ describes the number of radial nodes in the field). The nature and form of optically induced forces between particles trapped in the annular highintensity region of such a vortex are of special interest. Generally, experiments utilize two counter-propagating beams, to offset Maxwell-Bartoli forces and net rotation about the beam axis. When two or more particles are held in the LG trap, the distance $R$ between any pair of circumferentially adjacent particles $A$ and $B$ will usually be small compared to the radius of the optical trap, and the optically induced energy and force equations can be recast in terms of the angular displacement $\Delta \psi$; see Fig. $15(a)$. The general result (for arbitrary $p$ ) is as follows;

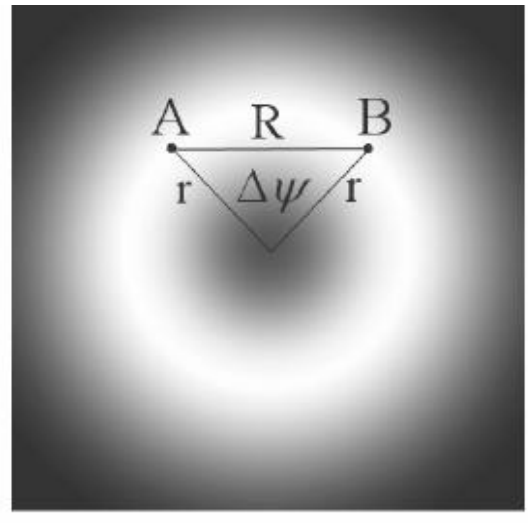

(a)

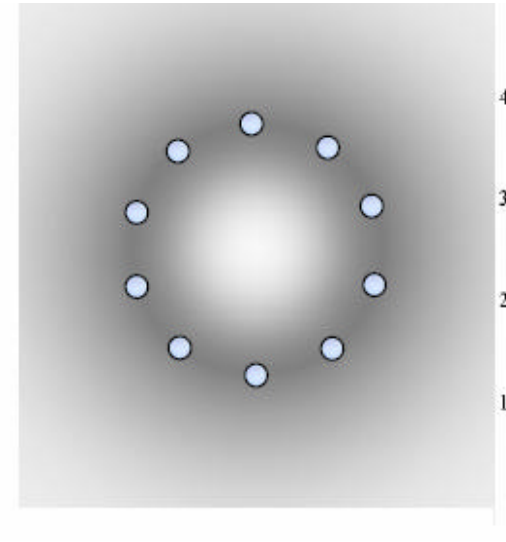

(b)

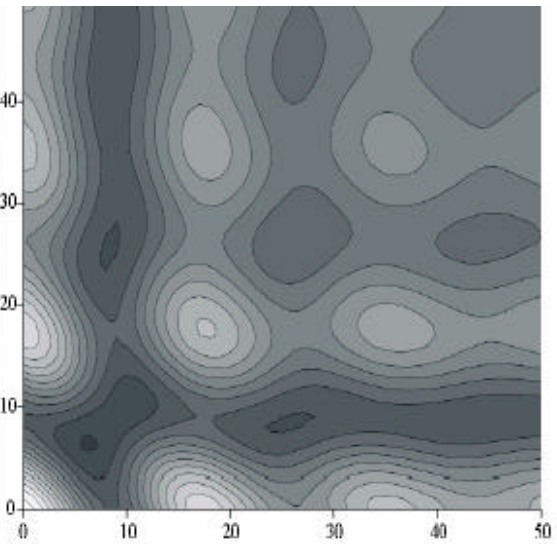

(c)

Fig 15. (a) Geometry of a particle pair in a Laguerre-Gaussian beam $(p=0) ;(b)$ Clustering of nanoparticles in an LG beam; (c) Contour graphs of $\Delta E_{A B C}^{0}$ against $\Delta \psi_{1}$ (x-axis) and $\Delta \psi_{2}(y$-axis) for three particles in an LG beam with $l=20$; lighter shading denotes higher values of $\Delta E_{A B C}^{0}$. Adapted from refs [143-145] 
$\Delta E_{A B C}^{0}=\left(\frac{I f_{l p}^{2} \alpha_{0}^{2}}{4 \pi \varepsilon_{0}^{2} c A_{p} R^{3}}\right)\left\{\cos ^{2} \phi\left(\cos k R+k R \sin k R-k^{2} R^{2} \cos k R\right)-2 \sin ^{2} \phi(\cos k R+k R \sin k R)\right\} \cos (l \Delta \psi)$.

where $f_{l p}$ and $A_{l p}$ are standard LG beam functions as defined in [146], and $\alpha_{0}$ is the polarizability of each spherical nanoparticle, $\phi$ is the angle between the polarization of the input radiation and $\boldsymbol{R}$, and $\Delta \psi$ is the azimuthal displacement angle. In the short-range region ( $k R \square 1$ ), the leading term of equation (20) is determined from Taylor series expansions of $\sin (k R)$ and $\cos (k R)$. By simple trigonometry, the result $\Delta E_{A B C}^{0}$ can be expressed as [143-145];

$$
\Delta E_{A B C}^{0}=\left[\frac{I f_{l p}^{2} \alpha_{0}^{2}\left(1-3 \sin ^{2} \phi\right)}{8 \sqrt{2} \pi \varepsilon_{0}^{2} r^{3} c A_{l p}}\right] \frac{\cos (l \Delta \psi)}{(\eta-\cos \Delta \psi)^{3 / 2}} .
$$

Here, $\eta$ is a damping factor whose introduction, in place of the unity that emerges from simple trigonometry precludes a singularity at $\Delta \psi=0$. The long-range (large ring radius) result has a considerably more complex asymptotic behaviour.

The result has a number of interesting features: (i) at $l=0$ - i.e. for a conventional Gaussian laser beam - a single energy minimum occurs at $\Delta \psi=180^{\circ}$, illustrating that the energetically most favourable position of the particles in the beam cross-section is where they are diametrically opposite each other, as might be expected; (ii) for odd values of $l>1$, only a local minimum (not the energetically most favourable) arises for this configuration; (iii) for even values of $l$, a local maximum occurs at $\Delta \psi=180^{\circ}$; (iv) generally, for $l$ ? 0 , there are $l$ angular minima and $(l-1)$ maxima. Additional features reflect the behaviour associated with increasing values of $l:(\mathrm{v})$ the number of positions for which the particle pair can be mutually trapped increases, becoming less energe tically favourable as the angular disposition increases towards diametric opposition, and; (vi) absolute minima are found at decreasing values of $\Delta \psi$ physically signifying a progression towards particle clustering.

To identify the possibilities for stable formations of more than two particles, as illustrated in Fig. $15(b)$, the two-particle analysis is readily extended to a system of three (or more) particles. In this case $\Delta E_{A B C}^{0}$ is determined by summing the pairwise laser-induced interactions between the three particles, employing variables $\Delta \psi_{1}$ and $\Delta \psi_{2}$ as the azimuthal displacements between particles $A-B$ and $B-C$ respectively. A typical contour plot of $\Delta E_{A B C}^{0}$ against $\Delta \psi_{1}$ and $\Delta \psi_{2}$ is exhibited in Fig. $15(c)$.

Interestingly, recent studies have begun to explore optical binding of nanoparticles linking potentially to some of the presented theoretical ideas. Svedberg and colleagues [147] have explored metallic nanoparticle pairs with applications for surface-enhanced Raman spectroscopy. In this work, they explored the behaviour of a system in which one trapped nanoparticle was brought a close vicinity of another adhered nanoparticle. The immobilised particle affected the potential energy landscape of the system and a second deep potential well for the trapped particle confinement was seen - a consequence of an optical binding 
effect. The trapped particle might "hop" between both potential wells. Dienerowitz et al. also explored nanoparticle behaviour within a Laguerre-Gaussian beam [148]. Particle confinement was mediated by scattering of the light field that was tuned below the plasmon resonance of the particles. The nanoparticles did not attract each other or accumulate as might be expected: in this case rather repulsion between the particles was observed with particles assuming diametrically opposite positions in the trap, as expected from the form of the binding interactions described above. Due to the optical angular momentum properties of the Laguerre-Gaussian beam, rotation was also observed as orbital angular momentum was transferred to the particles.

\subsection{Collapsing optically bound structures}

As has been shown, quantum electrodynamical studies of the optically induced inter-particle potential have revealed intricate features in the energy landscapes, indicating the possibility of optically inducing a variety of multi-particle structures, forces and torques using only a single pair of counter-propagating throughput beams [139]. If additional beams are introduced, the construction of significantly more complex three-dimensional particle configurations becomes possible. Indeed, even in the simple case of trapped atoms, stable configurations such as cubic structures can be established by the use of twisted beams in an optical molasses setup. The open structures thus formed are built on a scale where neither dispersion nor optically-induced forces are relevant [149].

In systems where multi-particle optical binding occurs, as discussed in Sect. 5.2, the introduction of additional beams offers other enticing prospects, including the deformation and dissipation of bound structures. Consider, for example, four plane waves in the setup shown in Fig. 16; from equation (12) an expression of the following form emerges for the inter-particle potential energy;[138]

$$
\Delta E=\left(\frac{k^{3} \alpha_{0}^{A} \alpha_{0}^{B}}{16 \pi^{2} c \varepsilon_{0}^{2}}\right)\left(\left[I_{s} D_{1}+2 I_{p} C_{1}\right] \frac{\cos k R}{k R}+\left[I_{s} D_{3}+2 I_{p} C_{3}\right]\left(\frac{\cos k R}{k^{3} R^{3}}+\frac{\sin k R}{k^{2} R^{2}}\right)\right) .
$$

where $C_{n}=\left(1-n \cos ^{2} \theta_{1}\right) \cos \left(k R \sin \theta_{1} \cos \theta_{2}\right)$ and $D_{n}=\left(2-n \sin ^{2} \theta_{1}\right) \cos \left(k R \cos \theta_{2}\right) ; I_{\mathrm{p}}$ and $I_{\mathrm{s}}$ are the irradiances of the primary and secondary counter-propagating beams, respectively. The two angles that feature in $C_{n}$ and $D_{n}$ are $\theta_{1}$, the angle between the displacement vector $\mathbf{R}$ and the $y$ axis, and $\theta_{2}$, the angle between the $\mathbf{R}$ projection on the $z x$ plane and the $x$ axis. In deriving (22), each pair of counter-propagating beams is assumed to have equal and opposite Poynting vectors - in practice their wavelengths will be offset to preclude holographic optical trapping.

Interesting features arise concerning the variations in inter-particle potential with the irradiances $I_{\mathrm{p}}$ and $I_{\mathrm{s}}$. In the near-field, (for particle separations $k R<<1$ ), and when $I_{s} \neq 2 I_{p}$, the dominant term in the expression for the pair energy may be written as; 


$$
\Delta E=\left[\frac{\left(2 I_{p}-I_{s}\right) \alpha_{0}^{A} \alpha_{0}^{B}}{16 \pi^{2} c \varepsilon_{0}{ }^{2}}\right] \frac{\left(1-3 \cos ^{2} \theta_{1}\right)}{R^{3}} .
$$

With $I_{s}>2 I_{p}$, such that the secondary beam exerts the principal optical trapping force directed along the $y$ axis, the first term in brackets in equation (23) is negative, and for any given particle separation a potential energy minimum will occur at $\theta_{1}= \pm \pi / 2$. This indicates that, under such conditions, an assembly of many particles can group in a lamellar form on the $x z$ plane of Fig. 16. Conversely, when $2 I_{p}>I_{s}$, the term in brackets is positive, and the potential energy minimum for a fixed particle separation is at $\theta_{1}=0$ or $\pm \pi$; in this case, a linear assembly will form along the $y$ axis.

The special case where $2 I_{p}=I_{s}$ merits careful attention, as the approximate limiting result given by equation (2) no longer applies. In applying the full result (22), the $R$-dependence of the $C_{n}$ and $D_{n}$ parameters therefore needs to be taken into account. As shown elsewhere [138], the inter-particle potential is then proportional to the inverse power of the particle separation; moreover it is always attractive. The result indicates that a system of particles, subjected to these conditions, will agglomerate. (A similar effect can be engineered in BoseEinstein condensates - see ref. [150]).

Consider the configuration shown in Fig. 16, when particles initially trapped in a conventional counter-propagating beam setup are subjected to secondary beams of increasing intensity. At first, with particles separated by small distances, the Casimir-Polder potential might be considered of considerable importance, its inverse sixth power distance dependence enabling a group of trapped particles to form a compact structure. However, the laser irradiances employed in optical trapping are usually high enough for the particles line up along the polarization direction, here the $y$ axis. By smoothly increasing the irradiance of the secondary laser (and also, thereby, the reflected field), the particle system is passively modified to a spherical form when the irradiance of the secondary field matches that of the counter-propagating beams. On further increase of the secondary beam irradiance, the sphere adapts a lamellar form transverse to the secondary field propagation direction. The initial structure can be recovered by reducing the irradiance of the secondary field.

When the particle separations are larger, in the order of magnitude of the laser wavelength, different arrays may be formed. When the secondary beam, $I_{s}$, is brought into play, and its irradiance increased, the most striking feature is the disappearance of the most prominent potential energy minimum originally located at $k R \sim 3$ (with $\theta_{1}=\pi / 2$ ), destabilizing a particle chain. The effect occurs at an intensity threshold $I_{s} \cong 1.2 I_{p}$. Physically, this signifies that a chain of particles which might originally extend in space over a distance of the order of micrometers collapses to a very much smaller structure, where the Casimir-Polder forces or short-range optical binding dominate. 


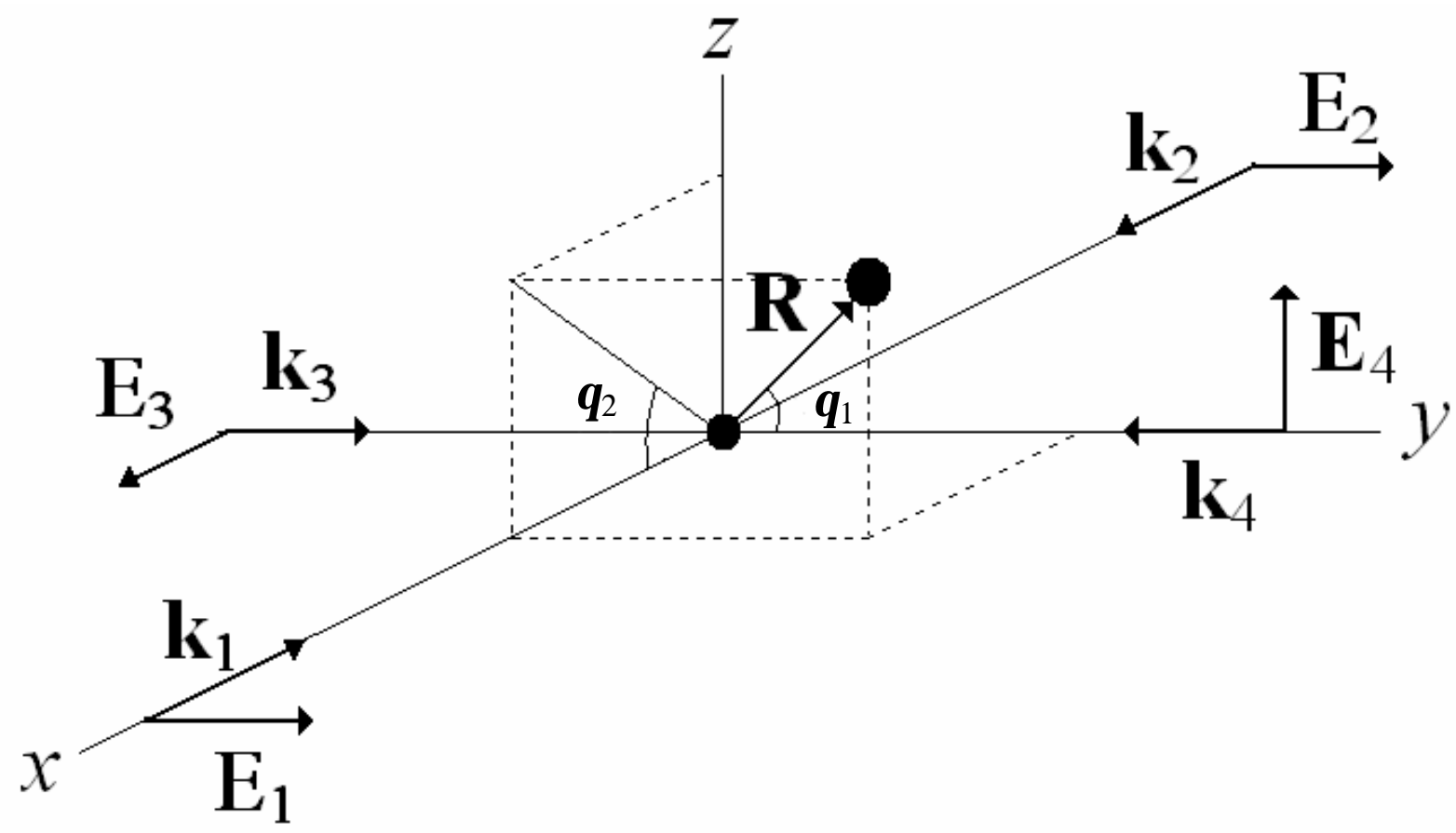

Fig 16. Pair of particles irradiated by counter-propagating beam pairs designated primary, $\left(\mathbf{E}_{1}, \mathbf{k}_{1}\right)$ and $\left(\mathbf{E}_{2}, \mathbf{k}_{2}\right)$, (standard optical binding) and secondary beams $\left(\mathbf{E}_{3}, \mathbf{k}_{3}\right.$ ) reflected with transverse polarization as $\left(\mathbf{E}_{4}, \mathbf{k}_{4}\right)$. The angle subtended by the interparticle displacement vector $\mathbf{R}$ on the $y$ axis is $\theta_{1}$, and the angle between the $\mathbf{R}$ projection on the $z x$ plane and the $x$ axis is $\theta_{2}$.

To elucidate the dynamics of optical binding with generality and rigour we have expanded upon the QED approach in detail, but naturally other approaches have been developed over the last few decades that have also proved accessible and useful for the experimental optical binding community. In terms of longitudinal optical binding, a paraxial optics approach has proved productive; this may be derived through approximations from a complete vectorial Maxwell theory. In this instance, the trapped particles refocus the incident optical fields and in turn this effects adjacent particles along the beam propagation direction. More specifically, the paraxial theory applies to particles in the Mie size regime whose diameter exceeds the optical wavelength, trapping beams that are not too tightly focused thereby allowing for a scalar paraxial treatment of the field propagation, allowing small refractiveindex mismatches between the bound spheres and surrounding medium: this means that backreflections may be largely neglected. With such assumptions, the paraxial theory accurately reproduces much data seen in longitudinal binding experiments. In comparison to the more general vector Maxwell theory the paraxial theory has calculational virtues in that it is considerably less computer-intensive, thereby allowing for treatment of longitudinal optical binding over spatial scales much larger than the wavelength. In recent work, paraxial approaches have been used to explain some of the phenomena observed in longitudinal optical binding such as chains in Gaussian and Bessel modes and bistability for two bound spheres (eg [107, 135]).

Other approaches have also proved popular: The coupled dipole approximation or discretedipole method (CDM) $[19,151]$ may be used to determine the scattering and absorption by arbitrarily shaped, inhomogeneous, anisotropic objects [152]. The technique is based on the 
decomposition of the object into many dipoles that are organized in an orthogonal grid with inter-dipole distances $d$. This distance is very small, thus replicating the overall object shape. The field in the region of each dipole can be considered uniform. Studies have shown that the coupled dipole approach is equivalent to a digitized Green's function method or volumeintegral equation formulation in the low-frequency limit [152]. Analogously to the paraxial approach, the CDM may lead to inaccuracies if the object refractive index difference between the particle and the surrounding medium. To capture such cases accurately, one may use a more complex description of the polarizability of the dipolar units (dipoles). In principle the CDM accuracy can be improved by increasing the number of dipoles $N$, but naturally this makes the method computationally very intensive. The CDM has been applied to a number of binding experiments. Recently it was applied to experimental studies of binding in two counter-propagating Bessel modes and predicted the observed behavior of long and short range creation of particle chains [153].

For large assemblies of homogenous spherical particles the generalized Lorenz-Mie theory or multiple scattering theory [154] came to the fore due to the relatively high precision and very high computational power efficiency. This approach extends the single-sphere Mie theory based on decomposition of the field quantities into a series of vector spherical harmonic functions. The expansion coefficients are obtained by solving a system of $2 \mathrm{NL}_{\max }\left(\mathrm{L}_{\max }+2\right)$ linear equations, where $\mathrm{N}$ is a number of the spheres and $\mathrm{L}_{\max }$ represents a truncation order that controls the precision of the results. This method was for example employed in theoretical predictions of photonics clusters formation [100] or to explain experimentally observed phenomena of large particle ensemble behaviour in evanescent standing wave geometries [155].

\section{6. $\quad$ Experiments for optical binding}

Experimentally it is now twenty years since the first experiments in optical binding. The two main experiments attributed with the birth of optical binding were performed in 1989 and 1990 by Burns et al. The first study [98] looked at the organisation of particles within a weakly focused light field incident upon a colloidal sample on a glass surface. These experiments explored what is now termed transverse optical binding where the incident light field is orthogonal to the direction of particle organisation. As well as first experimental data for transverse binding, the authors provided an analytical model for understanding the experiment. To interpret their experiments, they considered the incident field as a plane wave that in turn induced oscillating dipole moments in the particles, giving rise to scattering. The particle distance dictates the forces exerted, showing a dependence upon the phase of the scattered fields. An analytical expression for the interaction potential was determined by solving the coupled Maxwell-Lorentz equations for the system, in agreement with the QED equation (10).

More recent studies by Mohanty et al. explored such optical binding at near infra red wavelengths [106]. They investigated the motion of two polystyrene spheres (radius $\sim 150$ $\mathrm{nm}$ ) trapped in a near infra-red optical tweezers setup. The motion of the two particles was tracked for $\sim 25 \mathrm{~s}$ at video rate (25 frames per second) and found to be correlated. The histogram denoting the separation of the particles exhibited certain maxima, stable particle separations approximately correlating with multiples of the binding laser wavelength. Studies of the dependence on the plane of polarization of the interacting beam also gave good 
agreement with calculations based on considering the objects as dipoles interacting with an incoming plane wave.

In 2002, and six months later in 2003, two groups independently observed what is now termed longitudinal optical binding: here the particle organisation was achieved in one dimension in the counter-propagating trap geometry. Tatarkova et al. [5] used two weakly focused Gaussian beams similar to the original Ashkin geometry. They observed the formation of a string of trapped micron-sized particles in a system where the inter-particle spacing was much larger than the sphere size. The chains thus formed exhibited Brownian motion, but they were robust. Interference effects were avoided by the use of beams propagating in paths beyond their coherence length. The observations were attributed to a binding effect where in a simple model one could infer a given particle in the chain focused (or forward scattered) the incident field onto an adjacent particle. A naïve interpretation (without binding) might cause one to think all the particles would accumulate at trap centre, but this was not the case. The authors observed centre-of-mass motion and breathing mode type behaviour by appropriate manipulation of one of the trapping beams. By allowing more and more particles to build up within the array, the authors also identified the form of the trapping potential. At around the same time, Singer et al. secured analogous data for binding using the same geometry, but this time implemented with optical fibers and smaller particles [6]. Following the resurgence of general interest in optical binding, attention has refocused on the more widely studied system, longitudinal binding which we now progress to discuss.

\subsection{Longitudinal binding studies}

Subsequent to the first observations described above, Metzger et al. [135] performed a number of experiments to explore the dynamics of binding - including correlations in particle behaviour and visualization of the optical field. The researchers used a dual beam fibre trap to show the presence of bistability and hysteresis for two trapped spheres. In this study the formation of a 1D array of trapped micron-sized particles was observed as the fibre separation was varied. The observations were consistent with a theory treating the particles as Mie scatterers, using the Lorentz force formula in the paraxial regime, incorporating the focusing effect of each sphere. In the case of two spheres the system was naturally coupled, with an interdependency and interplay between the spheres due to light re-scattering. This, in turn, dictated the location of the equilibrium positions. Three points on the graph of force against separation indicate places where the force is zero and are thus positions of equilibrium: naturally only those with a negative slope are stable, leading to bistability. The origin of such bistability is the feedback inherent in the system. Altering the sphere separation changes the electromagnetic field distribution via a refocusing effect, which in turn modifies the forces on each sphere. Nonlinearity is not a necessary prerequisite for such bistability, and indeed such effects have been seen for example in the radiation pressure on an intracavity field on a moving mirror [156]. The experiment used two opposed single mode fibres to form the trap with powers up to $200 \mathrm{~mW}$; three micron diameter spheres were used in the study. The binding phenomenon was explored for variation in the fibre separation and the relative refractive index between the spheres and the medium. The system proved very sensitive to these parameters, and a clear observation of bistability, bifurcation of solutions and hysteresis when the fibre separation was changed adiabatically was observed. 


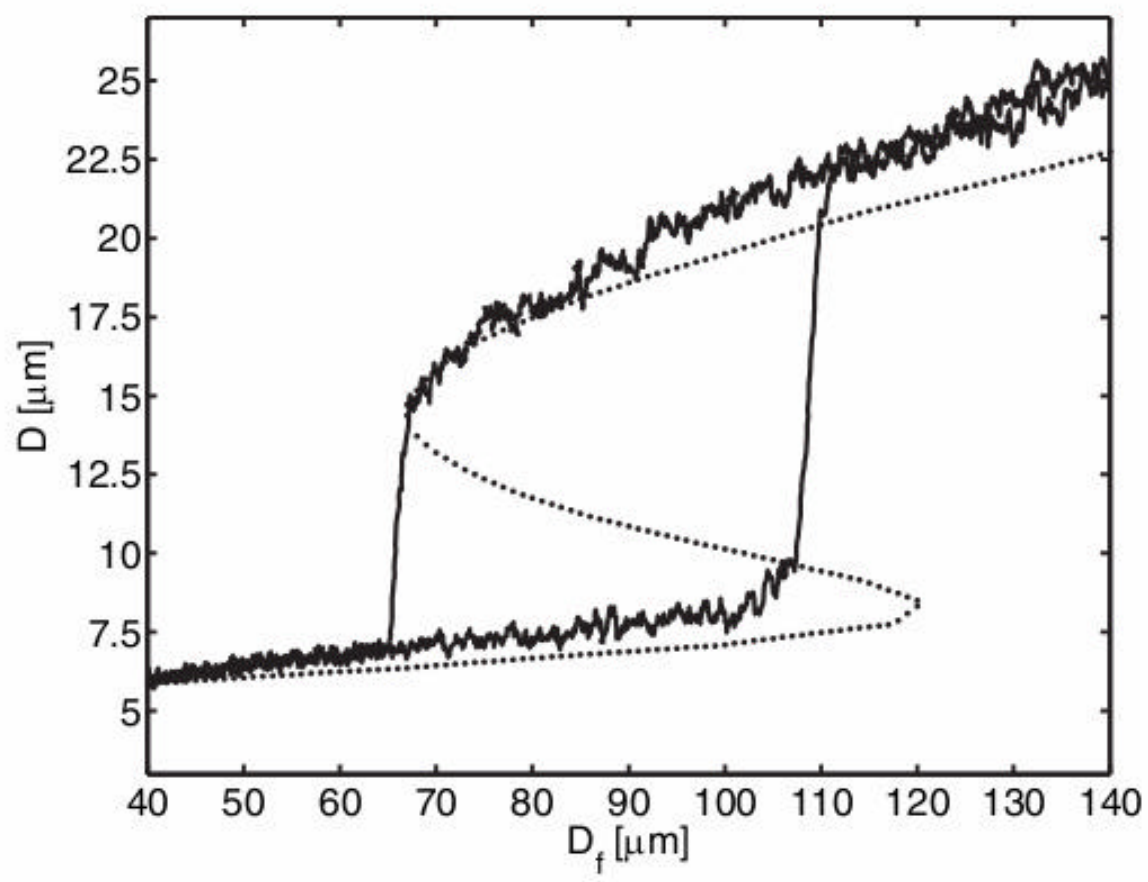

Fig 17: Multistability of particle separation in a fibre trap. With the varying distance between the fibre facets $D_{f}$ a separation between 2 trapped particles $\mathrm{D}$ changes. For a certain range of $D_{1}$ values there exist 2 stable configurations $D$, responsible for the observed hysteresis in the inter-particle separation while the fibre facets are moving towards or apart each other. Reprinted figure with permission from [135]. Copyright (2006) by the American Physical Society.

Subsequent studies by the same group developed a novel technique for an in situ visualisation of the scattered light field by a particle, making it possible to observe the particle position and gain a detailed intensity map of the light redistribution within the sample [157]. This was achieved by using fluorescein dye within the sample liquid and with ultrashort (femtosecond) pulses for the trapping and binding. The laser light was able to not only mimic the continuous-wave case for the binding (just like trapping, an average power effect) but an in situ optical field was seen due to two photon excitation in the liquid, giving an excellent signature of the light redistribution. 
A)
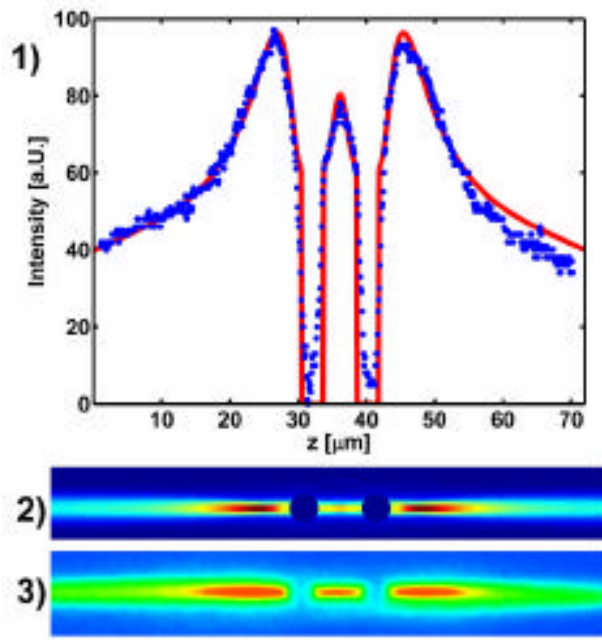

C)
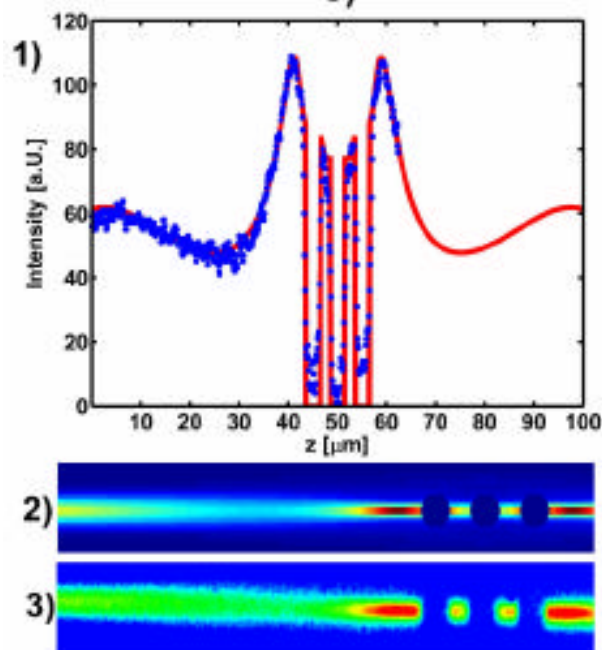

B)
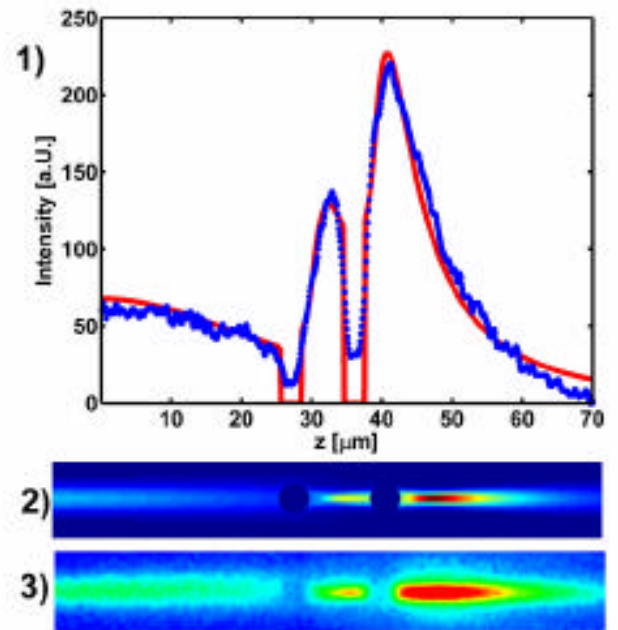

D)

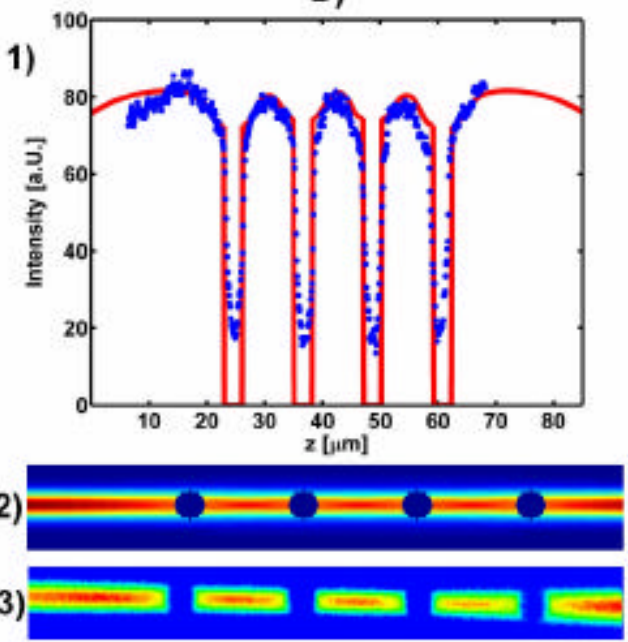

Fig. 18 Visualisation of optically bound arrays: A) Array of two $3 \mu \mathrm{m}$ spheres with a separation of $8 \mu \mathrm{m}$ with $? \mathrm{n}=0.06$. 1) On-axis intensity distribution showing the full waist separation of $72 \mu \mathrm{m}$ (blue - experimental data; red - theoretical prediction). 2) Theoretical simulation of diffraction pattern in a 2 sphere array, 3) False color image of two-photon fluorescence. B) Same array as in A) with left propagating field blocked, and for a time such that the sphere separation is $9 \mu \mathrm{m} .2$ ) Theoretical simulation of the diffraction pattern, 3) false color image of two-photon fluorescence beam coming from left side of picture. C) Array of three $3 \mu \mathrm{m}$ spheres with a separation of $5 \mu \mathrm{m}$ with $? \mathrm{n}=0.05$ and a waist separation of $100 \mu \mathrm{m}$. 1) On-axis intensity comparison between theory and experiment which is being cut off at $60 \mu \mathrm{m} .2$ ) and 3) theoretical and experimental images of diffraction pattern. D) Array of four $3 \mu \mathrm{m}$ sphere array with a separation of $12 \mu \mathrm{m}$ with $? \mathrm{n}=0.01$ with a waist separation of $85 \mu \mathrm{m}$. 1) On-axis intensity plot, 2) Theoretical image matching, 3) experimental false color image of two-photon fluorescence with right and left hand side of the beam being partly cut off. Reprinted figure and caption with permission from [108]. Copyright (2006) by the Optical Society of America.

The dependence upon each sphere in longitudinal binding lends itself to the interesting notion of correlated behaviour. This was explored by Metzger et al in their subsequent study [158], focusing on the motion of two microspheres in the presence of noise. The specific advance in this study was measurement of the decay time of the correlation functions of the centre of 
mass and relative normal modes of the bound matter system, allowing for an in situ determination of the optical restoring forces. The theoretical basis for this was an extension of studies performed by Meiners and Quake [159] and Bartlett et al. [160], including the hydrodynamic coupling between the particles. The authors developed the appropriate Langevin equations of motion for small amplitude sphere displacements, adding a cross-force term to account for the binding interaction. From this they derived the form of the correlation functions between the spheres. By experimentally measuring the normal mode decay times the researchers were able to access the direct and cross force components in situ. Both of these forces scale linearly with power, and so their ratio is power independent. The experiment employed dedicated particle tracking to make position measurements and infer the forces at play.

More recent experiments of longitudinal optical binding have had the aim of exploring interference effects and extending the range of the interaction. Gherardi et al. [161] developed a novel form of dual beam trap that used photonic crystal fibre that can potentially deliver multiple wavelengths at the same time rather than the more standard single mode fibre. This fibre was used to create optically bound arrays with broadband "white" light. The importance of this lay in the fact that such temporally incoherent light removed any artefacts in the binding from inter-particle interference that can create subsidiary maxima and minima in the binding process. This method will open up new studies in the future with multiple wavelengths for the binding process.

Karásek et al [134] developed the coupled dipole model (described earlier) to explore longitudinal binding. They explored how well their model mirrored the data of Metzger et al. [135] and then went onto explore long range optical binding. In their study they used two counter-propagating Bessel beams which are propagation-invariant or immune to diffraction over a given range. The use of these beams is attractive as they offer the potential to create very long $1 \mathrm{D}$ chains of bound particles, and might thus allow for a deeper understanding of free space self-assembly with light. In the experimental study two counter-propagating Bessel modes generated via axicon elements were used. The study showed a number of very new features, including the emergence of multistability due to interference effects between particles, with chains up to 200 microns long being formed [153].

In the last few years, optical force induced self-assembly and binding type behaviour has been observed in near-field optical trapping arrangements. Also, as in the experiments described already, the inherently coupled nature of the interactions driving these selfassembly processes give rise to nonlinear effects such as bistability and in particular modulational instability. These may be employed to generate particle arrays that are dynamically reconfigurable. Mellor and Bain [162] explored a near-field geometry in the Kretschmann configuration for trapping. They observed that large numbers of microscopic colloidal particles accumulated at a surface with counter-propagating interfering evanescent waves. Perhaps surprisingly, these particles formed highly ordered arrays with submicrometer precision. The lattice parameters were influenced by the polarization of the used optical field, the underlying interference standing-wave-like pattern generated, and the size of the trapped particles. Rotating the polarization of the trap by 90 degrees the researchers were able to see in real-time transitions between different lattice patterns namely checkerboard and hexagonal[163]. It was inferred that the ordering mechanism was intricately linked to optical binding. 


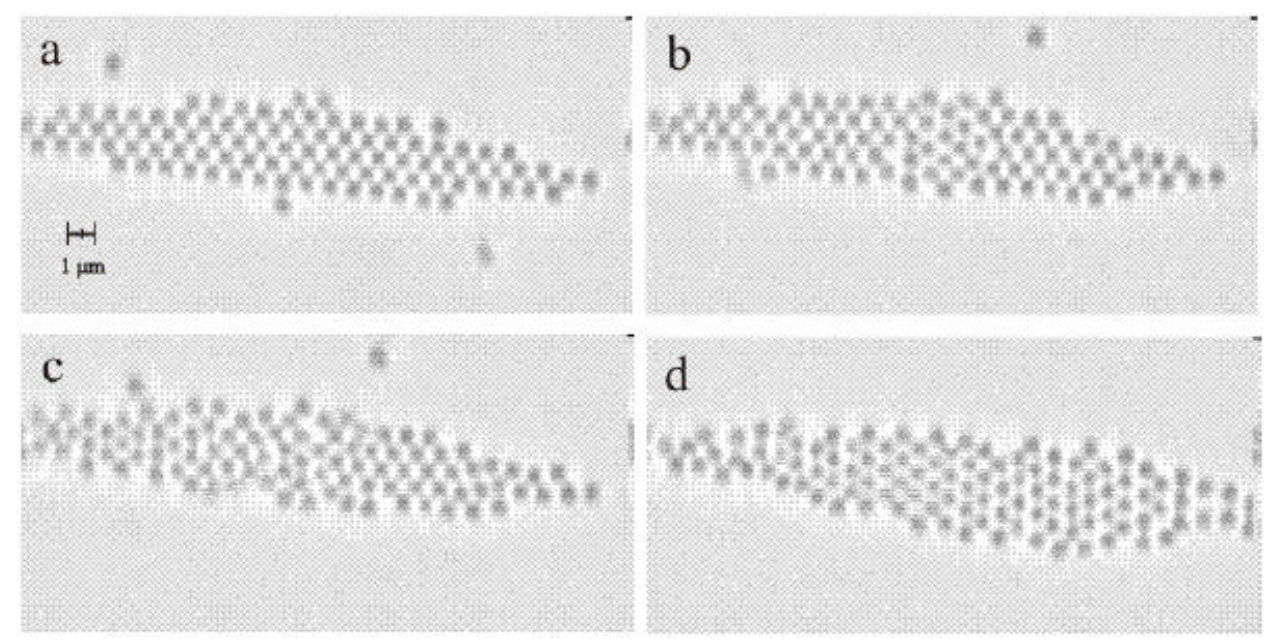

Fig.19. Observing self-organization by optical forces: binding on a surface. (a)-(d) Successive video frames showing the reorientation of a two-dimensional optically bound lattice when the polarization of the optical trap is switched from $s$ - to $p$ polarization; the lattice geometry is seen to change from a square lattice to a hexagonal lattice Reprinted with permission from [163] Copyright (2006) by the Optical Society of America.

The formation of 2D structures with sub-micron colloidal particles in surface (evanescent) traps was the further analysed theoretically and described by Taylor et al. Their studies showed that collective behaviour of larger numbers of optically trapped particles exhibits qualitative differences to that seen with small numbers. This was shown both with a computer model and in experimental results. The optical binding interaction can dominate as the number of particles in the structure is increased. Optical binding forces can be sufficiently large to overcome the optical landscape imposed by the interference fringes of the laser beams and impose a different, competing structure [155]. In this study, numerical modelling deployed a Generalized Lorenz-Mie Theory (GLMT) to determine the field and overall forces exerted on a group of spherical particles. In these calculations for spherical (and nonmagnetic) particles it proved sufficient to ignore any light re-scattered by the surface interface.
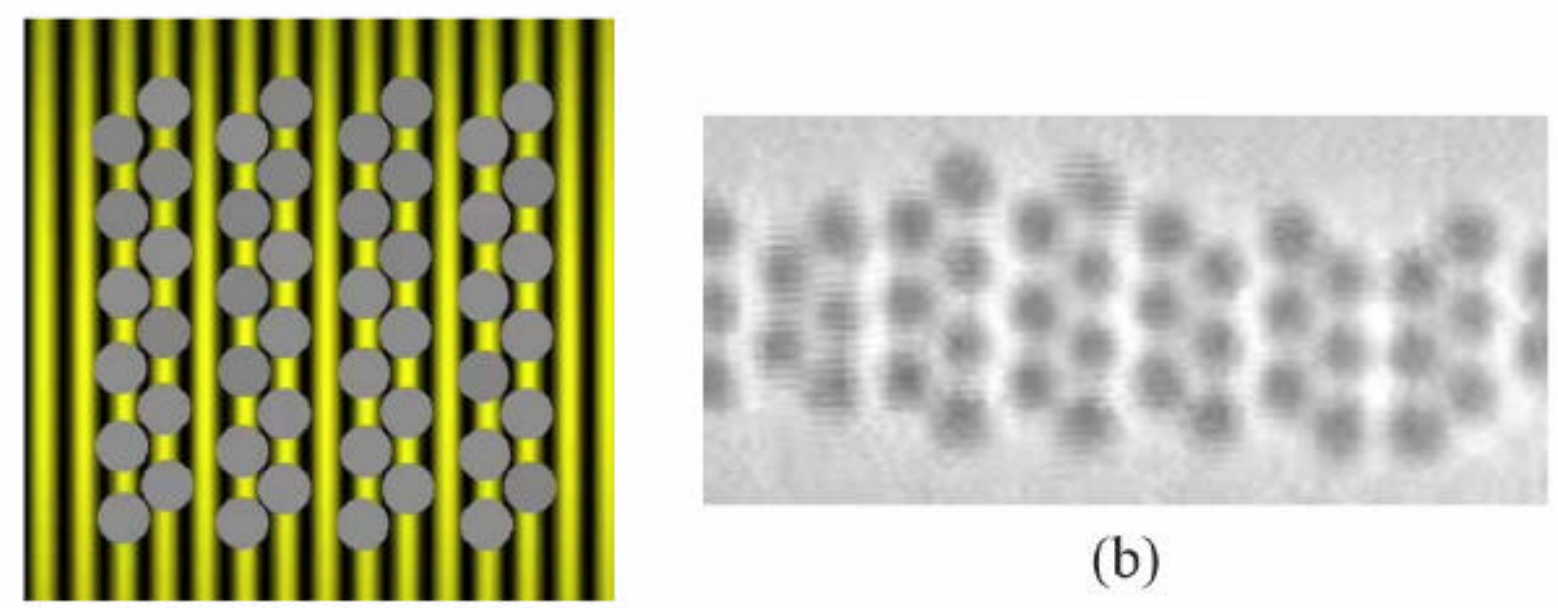

(b)

(a) 
Fig. 20. Examples of "broken hex" optically-bound structures with vacant fringes. (a) shows a snapshot from a Brownian dynamics simulation, and (b) shows an experimental image. In both cases the particle radius is $260 \mathrm{~nm}$. In the experimental image the vertical extent of the particle array is limited by the shape of the focused Gaussian beams. Reprinted figure and caption with permission from [155]. Copyright (2008) by the Optical Society of America.

In an analogous experimental arrangement, Reece et al. [164] also showed optically induced self-organization of microparticle arrays, but this time the incident field was un-patterned. Their system also used a dielectric resonator for enhancement of the evanescent field. High concentrations $(>0.1 \%$ solid) of sub-micrometer diameter particles were seen to collect at the centre of the overlap region of the beams. These colloidal particles formed regularly spaced linear arrays oriented along the direction of the incident beam axis with a lateral separation of several micrometers. The authors interpreted the behaviour of the colloidal arrays, formed parallel with the laser propagation-axis, as being linked to the break-up of the incident field into optical spatial solitons, the lateral spacing of the arrays being related to modulation instability of the colloidal system. In nonlinear media, optical spatial solitons (OSS) are spatially localized, non-diffracting modes that have gained much attention. They are the result of a balance between diffraction and self-phase modulation which results in selffocusing under intense illumination. Modulational instability (MI) was interpreted as the cause of this effect: for an incident field consisting of a plane wave, small wavefront perturbations may cause the optical field to break-up into periodic arrays of spatial optical solitons or more complex patterns.
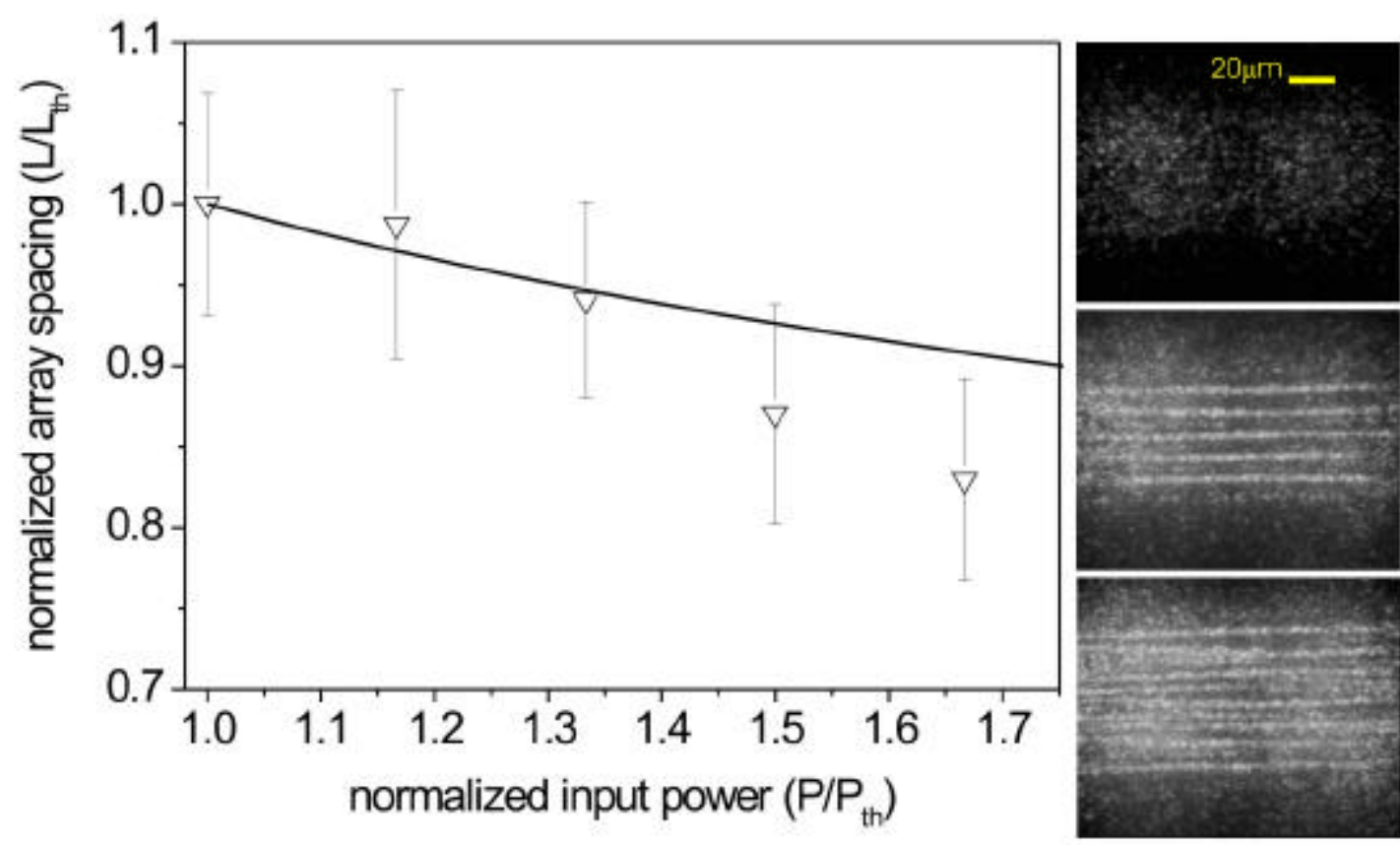

Fig. 21. Linear arrays of colloids observed at the surface of a prism acting as a dielectric resonator. The three pictures on the right illustrate array formations for different illumination intensities. Below the threshold soliton behaviour is not observed (top), even after extended periods of illumination. Just above threshold stable arrays of OSS are formed. The graph shows the period of array spacing as a function of the input power above threshold. The period $(L)$ is normalized with 
respect to its threshold value $\left(L_{t h}\right)$ and the input coupling power $(P)$ is normalized to the threshold power $\left(P_{t h}\right)$. The solid line indicates the variation as predicted by the continuum model. Reprinted figure with permission from [164]. Copyright (2007) by the American Physical Society.

The presence of both OSS and MI is suggestive of the presence of optical nonlinearity in the colloidal dispersion. In fact, such dispersions have been considered potential candidates for artificial optical nonlinear Kerr materials. Ashkin and co-workers indicated the potential of bulk colloidal suspensions to act as an artificial Kerr medium in a number of nonlinear optical experiments, including self-focusing, optical bistability and four-wave mixing [165]. The mechanism behind the nonlinear properties of these materials is an electrostrictive effect due to the optical gradient forces experienced by the dielectric particulates, which aggregate at regions of high intensity, thereby locally increasing the refractive index and leading to a self-phase modulation. In the experiments described by Reece et al. [164] the dielectric resonator was treated as a prism-coupled nonlinear waveguide where the colloidal dispersion acted as an artificial Kerr medium with a local nonlinear response (continuum approximation). This was sufficient to predict the main experimental observations; the presence of a threshold power for the onset of the array formation, the magnitude of the lateral array spacing and its power dependence. For a deeper understanding of the optical nonlinearity of the colloidal dispersions and its resulting effects the detailed granular nature must be considered, i.e. the non-local response. Recently, Conti et al. [166] developed a theoretical non-local model for the non-linear optical response of emulsions (including colloid dispersions) which accounts for microscopic particle-particle interactions using a static structure factor. However this is just one of a number of competing models which aim to describe the detailed dynamics of the nonlinear process.

\section{Future prospects for large scale self-assembly}

Larger scale controlled self-assembly of colloidal particles is certainly attainable in the near future. Optical fields can create and pattern particles in 2D and 3D thus forming various structures from microobjects at will. This has generated much interest in potentially creating templates for the nucleation of larger crystals: this may be used to realise cubic structures and other crystalline lattices. It is also important to emphasise that such templating is not restricted to colloids, but may be extended to biological cells. This in turn opens up the prospect of new studies in cell biology that includes templating for tissue generation and studies in cell differentiation.

Optical binding has revealed some interesting aspects of multi-particle interactions mediated by light. One also sees how this topic has revealed aspects of feedback due to the inherent interplay and coupling in these systems. This effect has been seen in transverse geometries and on surfaces where large numbers of particles have been organised. Indeed, checkerboard and hexagonal arrays have been seen on surfaces as well as organisation due to nonlinear effects. Numerical models are being developed to understand these effects. Longitudinal binding has seen immense advances since 2002 and numerical calculations have assisted the understanding between small numbers of objects. The inherent feedback in the system and interplay between the spheres has led to observations of bistability, multistability and chain formation. Numerical studies have been developed at size scales from the nanoscale to the 
microscale, and it is intriguing and instructive to compare these to emerging experiments to see the limitations and understand the exact results seen in experimental studies.

With the growth to maturity of both theory and experimental technique, the future for optical forces and their impact on multi-particle organisation is very promising: as we gain a deeper understanding and unravel the manner in which we can apply both multiplexed fields and exploit the self-organisation using optical binding, we will emerge with new tools for a variety of templates for new studies right across the natural sciences.

\section{Acknowledgements}

We thank the UK Engineering and Physical Sciences Research Council for funding. KD is a Royal Society-Wolfson Merit Award Holder.

\section{References}

[1] Anderson M H, Ensher J R, Matthews M R, Wieman C E and Cornell E A 1995 Observation of Bose-Einstein Condensation in a Dilute Atomic Vapor Science 269 198-201

[2] Wieman C E, Pritchard D E and Wineland D J 1999 Atom cooling, trapping, and quantum manipulation Reviews of Modern Physics 71 S253

[3] Ashkin A 1970 Acceleration and Trapping of Particles by Radiation Pressure Physical Review Letters 24 156-159

[4] Constable A, Kim J, Mervis J, Zarinetchi F and Prentiss M 1993 Demonstration of a Fiberoptic Light-Force Trap Optics Letters 18 1867-1869

[5] Tatarkova S A, Carruthers A E and Dholakia K 2002 One-dimensional optically bound arrays of microscopic particles Physical Review Letters 89283901

[6] Singer W, Frick M, Bernet S and Ritsch-Marte M 2003 Self-organized array of regularly spaced microbeads in a fiber-optical trap Journal of the Optical Society of America B-Optical Physics 20 1568-1574

[7] Ashkin A and Dziedzic J M 1974 Stability of Optical Levitation by Radiation Pressure Applied Physics Letters 24 586-588

[8] Gahagan K T and Swartzlander G A 1996 Optical vortex trapping of particles Optics Letters 21 827-829

[9] Sasaki K, Koshioka M, Misawa H, Kitamura N and Masuhara H 1992 Optical Trapping of a Metal-Particle and a Water Droplet by a Scanning Laser-Beam Applied Physics Letters 60 807-809

[10] Ashkin A, Dziedzic J M, Bjorkholm J E and Chu S 1986 Observation of a SingleBeam Gradient Force Optical Trap for Dielectric Particles Optics Letters 11 288-290

[11] Ashkin A 2006 Optical trapping and manipulation of neutral particles using lasers (Singapore; Hackensack, NJ: World Scientific)

[12] Dholakia K, Reece P and Gu M 2008 Optical micromanipulation Chemical Society Reviews 37 42-55

[13] Grier D G 2003 A revolution in optical manipulation Nature 424 810-816

[14] Jonáš A and Zemánek P 2008 Light at work: The use of optical forces for particle manipulation, sorting, and analysis Electrophoresis 29 4813-4851 
[15] Neuman K C and Block S M 2004 Optical trapping Review of Scientific Instruments 75 2787-2809

[16] Greene W M, Spjut R E, Bar-Ziv E, Sarofim A F and Longwell J P 1985

Photophoresis of Irradiated Spheres - Absorption Centers Journal of the Optical

Society of America B-Optical Physics 2 998-1004

[17] Gordon J P 1973 Radiation Forces and Momenta in Dielectric Media Physical Review A 8 14-21

[18] Chaumet P C and Nieto-Vesperinas M 2000 Time-averaged total force on a dipolar sphere in an electromagnetic field Optics Letters 25 1065-1067

[19] Draine B T 1988 The Discrete-Dipole Approximation And Its Application To Interstellar Graphite Grains Astrophysical Journal 333 848-872

[20] Svoboda K and Block S M 1994 Optical Trapping of Metallic Rayleigh Particles Optics Letters 19 930-932

[21] Hansen P M, Bhatia V K, Harrit N and Oddershede L 2005 Expanding the optical trapping range of gold nanoparticles Nano Letters 5 1937-1942

[22] Seol Y, Carpenter A E and Perkins T T 2006 Gold nanoparticles: enhanced optical trapping and sensitivity coupled with significant heating Optics Letters 31 2429-2431

[23] Dienerowitz M, Mazilu M and Dholakia K 2008 Optical manipulation of nanoparticles: a review Journal of Nanophotonics 2021875

[24] Pendleton A, Kundu S and Liang H 2009 Controlled synthesis of titanium nanochains using a template Journal of Nanoparticle Research 11 505-510

[25] Qin J Q, Wang X L, Jia D, Chen J, Fan Y X, Ding J and Wang H T 2009 FDTD approach to optical forces of tightly focused vector beams on metal particles Optics Express 17 8407-8416

[26] Saija R, Denti P, Borghese F, Maragó O M and Iati M A 2009 Optical trapping calculations for metal nanoparticles. Comparison with experimental data for Au and Ag spheres Optics Express 17 10231-10241

[27] Ashkin A 1992 Forces of a Single-Beam Gradient Laser Trap on a Dielectric Sphere in the Ray Optics Regime Biophysical Journal 61 569-582

[28] Novotny L and Hecht B 2006 Principles of nano-optics (Cambridge: Cambridge University Press)

[29] Jackson J D 1999 Classical electrodynamics (New York; Chichester: Wiley)

[30] Barton J P, Alexander D R and Schaub S A 1988 Internal and near-surface electromagnetic fields for a spherical particle irradiated by a focused laser beam Journal of Applied Physics 64 1632-1639

[31] Barton J P, Alexander D R and Schaub S A 1989 Theoretical Determination of NetRadiation Force and Torque for a Spherical-Particle Illuminated by a Focused LaserBeam Journal of Applied Physics 66 4594-4602

[32] Rohrbach A 2005 Stiffness of optical traps: Quantitative agreement between experiment and electromagnetic theory Physical Review Letters 95168102

[33] Rohrbach A and Stelzer E H K 2002 Trapping forces, force constants, and potential depths for dielectric spheres in the presence of spherical aberrations Applied Optics 41 2494-2507

[34] Guck J, Ananthakrishnan R, Mahmood H, Moon T J, Cunningham C C and Käs J 2001 The optical stretcher: A novel laser tool to micromanipulate cells Biophysical Journal 81 767-784

[35] Jess P R T, Garcés-Chávez V, Smith D, Mazilu M, Paterson L, Riches A, Herrington C S, Sibbett W and Dholakia K 2006 Dual beam fibre trap for Raman microspectroscopy of single cells Optics Express 14 5779-5791 
[36] Keen S and et al. 2007 Comparison of a high-speed camera and a quadrant detector for measuring displacements in optical tweezers Journal of Optics A: Pure and Applied Optics 9 S264

[37] Berg-Sørensen K and Flyvbjerg H 2004 Power spectrum analysis for optical tweezers Review of Scientific Instruments 75 594-612

[38] Berg-Sørensen K, Oddershede L and Flyvbjerg H 2004 Optical tweezers as a tool of precision: Single-molecule mobility as case study. In: Conference on Imaging, Manipulation and Analysis of Biomolecules, Cells and Tissues II, ed D V Nicolau et al. (San Jose, CA pp 64-74

[39] Brau R R and et al. 2007 Passive and active microrheology with optical tweezers Journal of Optics A: Pure and Applied Optics 9 S103

[40] Svoboda K, Schmidt C F, Schnapp B J and Block S M 1993 Direct Observation of Kinesin Stepping by Optical Trapping Interferometry Nature 365 721-727

[41] Abbondanzieri E A, Greenleaf W J, Shaevitz J W, Landick R and Block S M 2005 Direct observation of base-pair stepping by RNA polymerase Nature 438 460-465

[42] La Porta A and Wang M D 2004 Optical Torque Wrench: Angular Trapping, Rotation, and Torque Detection of Quartz Microparticles Physical Review Letters 92 190801

[43] Volpe G, Volpe G and Petrov D 2007 Brownian motion in a nonhomogeneous force field and photonic force microscope Physical Review E (Statistical, Nonlinear, and Soft Matter Physics) 76061118

[44] Friese M E J, Rubinsztein-Dunlop H, Gold J, Hagberg P and Hanstorp D 2001 Optically driven micromachine elements Applied Physics Letters 78 547-549

[45] Galajda P and Ormos P 2001 Complex micromachines produced and driven by light Applied Physics Letters 78 249-251

[46] Terray A, Oakey J and Marr D W M 2002 Microfluidic control using colloidal devices Science 296 1841-1844

[47] Bechinger C, Brunner M and Leiderer P 2001 Phase behavior of two-dimensional colloidal systems in the presence of periodic light fields Physical Review Letters $\mathbf{8 6}$ 930-933

[48] Korda P T, Spalding G C and Grier D G 2002 Evolution of a colloidal critical state in an optical pinning potential landscape Physical Review B 66024504

[49] van Dillen T, van Blaaderen A and Polman A 2004 Shaping colloidal assemblies Materials Today 7 40-46

[50] Burns M M, Fournier J M and Golovchenko J A 1990 Optical Matter- Crystallization and Binding in Intense Optical Fields Science 249 749-754

[51] Chiou A E, Wang W, Sonek G J, Hong J and Berns M W 1997 Interferometric optical tweezers Optics Communications 133 7-10

[52] Zemánek P, Jonáš A, Sramek L and Liska M 1998 Optical trapping of Rayleigh particles using a Gaussian standing wave Optics Communications 151 273-285

[53] Cižmár T, Garcés-Chávez V, Dholakia K and Zemánek P 2005 Optical conveyor belt for delivery of submicron objects Applied Physics Letters 86174101

[54] Cižmár T, Šiler M, Šerý M, Zemánek P, Garcés-Chávez V and Dholakia K 2006 Optical sorting and detection of submicrometer objects in a motional standing wave Physical Review B 74035105

[55] Garcés-Chávez V, Dholakia K and Spalding G C 2005 Extended-area optically induced organization of microparticies on a surface Applied Physics Letters $\mathbf{8 6}$ 031106 
[56] Garcés-Chávez V, Quidant R, Reece P J, Badenes G, Torner L and Dholakia K 2006 Extended organization of colloidal microparticles by surface plasmon polariton excitation Physical Review B 73085417

[57] Righini M, Zelenina A S, Girard C and Quidant R 2007 Parallel and selective trapping in a patterned plasmonic landscape Nature Physics 3 477-480

[58] Birkbeck A L, Flynn R A, Ozkan M, Song D Q, Gross M and Esener S C 2003 VCSEL Arrays as micromanipulators in chip-based biosystems Biomedical Microdevices 5 47-54

[59] Flynn R A, Birkbeck A L, Gross M, Ozkan M, Shao B, Wang M M and Esener S C 2002 Parallel transport of biological cells using individually addressable VCSEL arrays as optical tweezers Sensors and Actuators B-Chemical 87 239-243

[60] Shao B, Zlatanovic S, Ozkan M, Birkbeck A L and Esener S C 2006 Manipulation of microspheres and biological cells with multiple agile VCSEL traps Sensors and Actuators B-Chemical 113 866-874

[61] Fällman E and Axner O 1997 Design for fully steerable dualtrap optical tweezers Applied Optics 36 2107-2113

[62] Sasaki K, Koshioka M, Misawa H, Kitamura N and Masuhara H 1991 Patternformation and flow-control of fine particles by laser-scanning micromanipulation Optics Letters 16 1463-1465

[63] Mio C, Gong T, Terray A and Marr D W M 2000 Design of a scanning laser optical trap for multiparticle manipulation Review of Scientific Instruments $712196-2200$

[64] Visscher K, Gross S P and Block S M 1996 Construction of multiple-beam optical traps with nanometer-resolution position sensing IEEE Journal of Selected Topics in Quantum Electronics 2 1066-1076

[65] Vossen D L J, van der Horst A, Dogterom M and van Blaaderen A 2004 Optical tweezers and confocal microscopy for simultaneous three-dimensional manipulation and imaging in concentrated colloidal dispersions Review of Scientific Instruments $\mathbf{7 5}$ 2960-2970

[66] Moffitt J R, Chemla Y R, Smith S B and Bustamante C 2008 Recent advances in optical tweezers Annual Review of Biochemistry 77 205-228

[67] Dame R T, Noom M C and Wuite G J L 2006 Bacterial chromatin organization by HNS protein unravelled using dual DNA manipulation Nature 444 387-390

[68] Guilford W H, Tournas J A, Dascalu D and Watson D S 2004 Creating multiple timeshared laser traps with simultaneous displacement detection using digital signal processing hardware Analytical Biochemistry 326 153-166

[69] Valentine M T, Guydosh N R, Gutiérrez-Medina B, Fehr A N, Andreasson J O and Block S M 2008 Precision steering of an optical trap by electro-optic deflection Optics Letters 33 599-601

[70] Fournier J M, Burns M M and Golovchenko J A 1995. Writing diffractive structures by optical trapping Proc. SPIE, 2406 101-111

[71] He H, Friese M E J, Heckenberg N R and Rubinsztein-Dunlop H 1995 Direct Observation Of Transfer Of Angular-Moment um To Absorptive Particles From A Laser-Beam With A Phase Singularity Physical Review Letters 75 826-829

[72] He H, Heckenberg N R and Rubinsztein-Dunlop H 1995 Optical-Particle Trapping with Higher-Order Doughnut Beams Produced Using High-Efficiency ComputerGenerated Holograms Journal of Modern Optics 42 217-223

[73] Dufresne E R and Grier D G 1998 Optical tweezer arrays and optical substrates created with diffractive optics Review of Scientific Instruments 69 1974-1977

[74] Curtis J E, Koss B A and Grier D G 2002 Dynamic holographic optical tweezers Optics Communications 207 169-175 
[75] Dufresne E R, Spalding G C, Dearing M T, Sheets S A and Grier D G 2001 Computer-generated holographic optical tweezer arrays Review of Scientific Instruments 72 1810-1816

[76] Grier D G and Roichman Y 2006 Holographic optical trapping Applied Optics 45 880-887

[77] Hossack W J, Theofanidou E, Crain J, Heggarty K and Birch M 2003 High-speed holographic optical tweezers using a ferroelectric liquid crystal microdisplay Optics Express 11 2053-2059

[78] Leach J, Sinclair G, Jordan P, Courtial J, Padgett M J, Cooper J and Laczik Z J 2004 3D manipulation of particles into crystal structures using holographic optical tweezers Optics Express 12 220-226

[79] Lee S H and Grier D G 2005 Robustness of holographic optical traps against phase scaling errors Optics Express 13 7458-7465

[80] Liesener J, Reicherter M, Haist T and Tiziani H J 2000 Multi-functional optical tweezers using computer-generated holograms Optics Communications 185 77-82

[81] Martín-Badosa E, Montes-Usategui M, Carnicer A, Andilla J, Pleguezuelos E and Juvells I 2007 Design strategies for optimizing holographic optical tweezers set- ups Journal of Optics A: Pure and Applied Optics 9 S267-S277

[82] Melville H, Milne G F, Spalding G C, Sibbett W, Dholakia K and McGloin D 2003 Optical trapping of three-dimensional structures using dynamic holograms Optics Express 11 3562-3567

[83] Montes-Usategui M, Pleguezuelos E, Andilla J and Martín-Badosa E 2006 Fast generation of holographic optical tweezers by random mask encoding of Fourier components Optics Express 14 2101-2107

[84] Polin M, Ladavac K, Lee S H, Roichman Y and Grier D G 2005 Optimized holographic optical traps Optics Express 13 5831-5845

[85] Schmitz C H J, Spatz J P and Curtis J E 2005 High-precision steering of multiple holographic optical traps Optics Express 13 8678-8685

[86] Sinclair G, Jordan P, Leach J, Padgett M J and Cooper J 2004 Defining the trapping limits of holographical optical tweezers Journal of Modern Optics 51 409-414

[87] Sinclair G, Leach J, Jordan P, Gibson G, Yao E, Laczik Z J, Padgett M J and Courtial J 2004 Interactive application in holographic optical tweezers of a multi-plane Gerchberg-Saxton algorithm for three-dimensional light shaping Optics Express 12 $1665-1670$

[88] Whyte G and Courtial J 2005 Experimental demonstration of holographic threedimensional light shaping using a Gerchberg-Saxton algorithm New Journal of Physics 7117

[89] Jesacher A, Furhapter S, Bernet S and Ritsch-Marte M 2004 Diffractive optical tweezers in the Fresnel regime Optics Express 12 2243-2250

[90] Cižmár T, Kollárová V, Tsampoula X, Gunn-Moore F, Sibbett W, Bouchal Z and Dholakia K 2008 Generation of multiple Bessel beams for a biophotonics workstation Optics Express 16 14024-14035

[91] Paterson L, Papagiakoumou E, Milne G, Garcés-Chávez V, Tatarkova S A, Sibbett W, Gunn-Moore F J, Bryant P E, Riches A C and Dholakia K 2005 Light-induced cell separation in a tailored optical landscape Applied Physics Letters 87123901

[92] McLeod E and Arnold C B 2008 Subwavelength direct-write nanopatterning using optically trapped microspheres Nature Nanotechnology 3 413-417

[93] Eriksen R L, Daria V R and Glückstad J 2002 Fully dynamic multiple-beam optical tweezers Optics Express 10 597-602 
[94] Rodrigo P J, Perch-Nielsen I R, Alonzo C A and Glückstad J 2006 GPC-based optical micromanipulation in 3D real-time using a single spatial light modulator Optics Express 14 13107-13112

[95] Dam J S, Rodrigo P J, Perch-Nielsen I R, Alonzo C A and Glückstad J 2007 Computerized "drag-and-drop" alignment of GPC-based optical micromanipulation system Optics Express 15 1923-1931

[96] Atkins P W and Paula J d 2006 Atkins' physical chemistry (Oxford: Oxford University Press)

[97] Thirunamachandran T 1980 Intermolecular interaction in the presence of an intense radiation- field Molecular Physics 40 393-399

[98] Burns M M, Fournier J M and Golovchenko J A 1989 Optical Binding Physical Review Letters 63 1233-1236

[99] Whitesides G 1990 What Will Chemistry Do in the Next 20 Years Angewandte Chemie-International Edition in English 29 1209-1218

[100] Ng J, Lin Z F, Chan C T and Sheng P 2005 Photonic clusters formed by dielectric microspheres: Numerical simulations Physical Review B 72085130

[101] Milonni P W and Smith A 1996 Van der Waals dispersion forces in electromagnetic fields Physical Review A 53 3484-3489

[102] Depasse F and Vigoureux J M 1994 Optical Binding Force between Two Rayleigh Particles Journal of Physics D-Applied Physics 27 914-919

[103] Milonni P W and Shih M L 1992 Source Theory of the Casimir Force Physical Review A 45 4241-4253

[104] Chaumet P C and Nieto-Vesperinas M 2001 Optical binding of particles with or without the presence of a flat dielectric surface Physical Review B 64035422

[105] Nieto-Vesperinas M, Chaumet P C and Rahmani A 2004 Near-field photonic forces Philosophical Transactions of the Royal Society a-Mathematical Physical and Engineering Sciences 362 719-737

[106] Mohanty S K, Andrews J T and Gupta P K 2004 Optical binding between dielectric particles Optics Express 12 2746-2753

[107] McGloin D, Carruthers A E, Dholakia K and Wright E M 2004 Optically bound microscopic particles in one dimension Phys Rev E Stat Nonlin Soft Matter Phys 69 021403

[108] Metzger N K, Wright E M, Sibbett W and Dholakia K 2006 Visualization of optical binding of microparticles using a femtosecond fiber optical trap Opt. Express 14 3677-3687

[109] Guillon M and Stout B 2008 Optical trapping and binding in air: Imaging and spectroscopic analysis Physical Review A (Atomic, Molecular, and Optical Physics) 77023806

[110] Marchington R F, Mazilu M, Kuriakose S, Garcés-Chávez V, Reece P J, Krauss T F, Gu M and Dholakia K 2008 Optical deflection and sorting of microparticles in a nearfield optical geometry Optics Express 16 3712-3726

[111] Andrews D L and Bradshaw D S 2004 Virtual photons, dipole fields and energy transfer: a quantum electrodynamical approach European Journal of Physics 25 845858

[112] Casimir H B G and Polder D 1948 The influence of retardation on the London-Van der Waals forces Physical Review 73 360-372

[113] Milonni P W 1994 The quantum vacuum: an introduction to quantum electrodynamics (Boston, Mass.; London: Academic Press)

[114] Andrews D L and Dávila Romero L C 2001 Conceptualization of the Casimir effect European Journal of Physics 22 447-451 
[115] Power E A 2001 Casimir-Polder potential from first principles European Journal of Physics 22 453-461

[116] Maclay G J, Fearn H and Milonni P W 2001 Of some theoretical significance: implications of Casimir effects European Journal of Physics 22 463-469

[117] Alligood B W and Salam A 2007 On the application of state sequence diagrams to the calculation of the Casimir-Polder potential Molecular Physics 105 395-404

[118] Capasso F, Munday J N, Iannuzzi D and Chan H B 2007 Casimir forces and quantum electrodynamical torques: Physics and nanomechanics IEEE Journal of Selected Topics in Quantum Electronics 13 400-414

[119] Altland A and Simons B 2006 Condensed matter field theory (Cambridge; New York: Cambridge University Press)

[120] Bradshaw D S and Andrews D L 2006 Optically induced forces and torques: Interactions between nanoparticles in a laser beam (vol 72, 033816, 2005) Physical Review A 73039903

[121] Dávila Romero L C and Andrews D L 2008 Structured Light and Its Applications: An Introduction to Phase-Structured Beams and Nanoscale Optical Forces, ed D L Andrews (Burlington MA: Academic Press) pp 79-105

[122] Salam A 2006 Intermolecular interactions in a radiation field via the method of induced moments Physical Review A 73013406

[123] Daniels G J, Jenkins R D, Bradshaw D S and Andrews D L 2003 Resonance energy transfer: The unified theory revisited Journal of Chemical Physics 119 2264-2274

[124] Rodríguez J and Andrews D L 2009 Inter-particle interaction induced by broadband radiation Optics Communications 282 2267-2269

[125] Rodríguez J, Dávila Romero L C and Andrews D L 2008 Optical binding in nanoparticle assembly: Potential energy landscapes Physical Review A 78043805

[126] Andrews D L and Bradshaw D S 2005 Laser-induced forces between carbon nanotubes Optics Letters 30 783-785

[127] Guo L, Liang J, Dong S, Xu Z and Zhao Q 2004 Property of carbon nanotube tip for surface topography characterization Applied Surface Science 228 53-56

[128] Grzegorczyk T M, Kemp B A and Kong J A 2006 Stable optical trapping based on optical binding forces Physical Review Letters 96113903

[129] Grzegorczyk T M, Kemp B A and Kong J A 2006 Trapping and binding of an arbitrary number of cylindrical particles in an in-plane electromagnetic field Journal of the Optical Society of America a-Optics Image Science and Vision 23 2324-2330

[130] Andrews D L and Allcock P 2002 Optical harmonics in molecular systems (Weinheim ; [Great Britain]: Wiley-VCH)

[131] Chen F, Klimchitskaya G L, Mostepanenko V M and Mohideen U 2007 Demonstration of optically modulated dispersion forces Optics Express 15 4823-4829

[132] Deniz A A, Mukhopadhyay S and Lemke E A 2008 Single-molecule biophysics: at the interface of biology, physics and chemistry Journal of the Royal Society Interface 5 15-45

[133] Karásek V, Brzobohatý O and Zemánek P 2009 Longitudinal optical binding of several spherical particles studied by the coupled dipole method Journal of Optics aPure and Applied Optics 119

[134] Karásek V, Dholakia K and Zemánek P 2006 Analysis of optical binding in one dimension Applied Physics B-Lasers and Optics 84 149-156

[135] Metzger N K, Dholakia K and Wright E M 2006 Observation of bistability and hysteresis in optical binding of two dielectric spheres Physical Review Letters 96 068102 
[136] Rodríguez J, Dávila Romero L C and Andrews D L 2007 Optically induced potential energy landscapes Journal of Nanophotonics 17

[137] Pedraza A J, Fowlkes J D, Blom D A and Meyer H M 2002 Laser-induced nanoparticle ordering Journal of Materials Research 17 2815-2822

[138] Andrews D L and Rodríguez J 2008 Collapse of optical binding under secondary irradiation Optics Letters 33 1830-1832

[139] Dávila Romero L C, Rodríguez J and Andrews D L 2008 Electrodynamic mechanism and array stability in optical binding Optics Communications $\mathbf{2 8 1}$ 865-870

[140] Mohanty S and Mohanty K 2007 Single fiber optical tweezers for manipulation of microscopic objects In: Conference on Imaging, Manipulation, and Analysis of Biomolecules, Cells, and Tissues V, ed D L Farkas, et al. (San Jose, CA: Spie-Int Soc Optical Engineering) p 644116

[141] Guillon M 2006 Field enhancement in a chain of optically bound dipoles Opt. Express 14 3045-3055

[142] Andrews D L 2008 Structured Light and Its Applications: An Introduction to PhaseStructured Beams and Nanoscale Optical Forces (Burlington MA: Academic Press)

[143] Bradshaw D S and Andrews D L 2005 Interactions between spherical nanoparticles optically trapped in Laguerre-Gaus sian modes Optics Letters 30 3039-3041

[144] Bradshaw D S and Andrews D L 2005 Optical forces between dielectric nanoparticles in an optical vortex. In: Nanomanipulation with Light, ed D L Andrews (San José, CA, USA: SPIE) pp 87-95

[145] Bradshaw D S and Andrews D L 2006 Optical ordering of nanoparticles trapped by Laguerre-Gaussian laser modes In: Nanomanipulation with Light II, ed D L Andrews (San José, CA, USA: SPIE) pp G1310-G1310

[146] Allen L, Padgett M J and Babiker M 1999 The orbital angular momentum of light Progress in Optics 39 291-372

[147] Svedberg F, Li Z P, Xu H X and Käll M 2006 Creating hot nanoparticle pairs for surface-enhanced Raman spectroscopy through optical manipulation Nano Letters 6 2639-2641

[148] Dienerowitz M, Mazilu M, Reece P J, Krauss T F and Dholakia K 2008 Optical vortex trap for resonant confinement of metal nanoparticles Optics Express 16 49914999

[149] Carter A R, Babiker M, Al-Amri M and Andrews D L 2006 Generation of microscale current loops, atom rings, and cubic clusters using twisted optical molasses Physical Review A 73 021401(R)

[150] O'Dell D, Giovanazzi S, Kurizki G and Akulin V M 2000 Bose-Einstein condensates with $1 / \mathrm{r}$ interatomic attraction: Electromagnetically induced "gravity" Physical Review Letters 84 5687-5690

[151] Purcell E M and Pennypacker C R 1973 Scattering and Absorption of Light by Nonspherical Dielectric Grains Astrophysical Journal 186 705-714

[152] Draine B T and Flatau P J 1994 Discrete-Dipole Approximation for Scattering Calculations Journal of the Optical Society of America a-Optics Image Science and Vision 11 1491-1499

[153] Karásek V, Cižmár T, Brzobohatý O, Zemánek P, Garcés-Chávez V and Dholakia K 2008 Long-range one-dimensional longitudinal optical binding Physical Review Letters 101143601

[154] Xu Y-1 1995 Electromagnetic scattering by an aggregate of spheres Appl. Opt. 34 4573-4588

[155] Taylor J M, Wong L Y, Bain C D and Love G D 2008 Emergent properties in optically bound matter Optics Express 16 6921-6929 
[156] Dorsel A, McCullen J D, Meystre P, Vignes E and Walther H 1983 Optical Bistability and Mirror Confinement Induced by Radiation Pressure Physical Review Letters $\mathbf{5 1}$ $1550-1553$

[157] Metzger N K, Wright E M and Dholakia K 2006 Theory and simulation of the bistable behaviour of optically bound particles in the Mie size regime New Journal of Physics 8139

[158] Metzger N K, Marchington R F, Mazilu M, Smith R L, Dholakia K and Wright E M 2007 Measurement of the Restoring Forces Acting on Two Optically Bound Particles from Normal Mode Correlations Physical Review Letters 98068102

[159] Meiners J C and Quake S R 1999 Direct measurement of hydrodynamic cross correlations between two particles in an external potential Physical Review Letters 82 2211-2214

[160] Bartlett P, Henderson S I and Mitchell S J 2001 Measurement of the hydrodynamic forces between two polymer-coated spheres Philosophical Transactions of the Royal Society a-Mathematical Physical and Engineering Sciences 359 883-893

[161] Gherardi D M, Carruthers A E, Cižmár T, Wright E M and Dholakia K 2008 A dual beam photonic crystal fiber trap for microscopic particles Applied Physics Letters $\mathbf{9 3}$ 041110

[162] Mellor C D and Bain C D 2006 Array formation in evanescent waves Chemphyschem 7 329-332

[163] Mellor C D, Fennerty T A and Bain C D 2006 Polarization effects in optically bound particle arrays Optics Express 14 10079-10088

[164] Reece P J, Wright E M and Dholakia K 2007 Experimental observation of modulation instability and optical spatial soliton arrays in soft condensed matter Physical Review Letters 98203902

[165] Ashkin A, Dziedzic J M and Smith P W 1982 Continuous-wave self-focusing and self-trapping of light in artificial Kerr media Opt. Lett. 7 276-278

[166] Conti C, Ruocco G and Trillo S 2005 Optical spatial solitons in soft matter Physical Review Letters 95183902 